\title{
Multi-omics of the esophageal microenvironment identifies signatures associated with progression of Barrett's esophagus
}

\author{
Nandan P. Deshpande1, Stephen M. Riordan², Claire J. Gorman³ , Shaun Nielsen³, Tonia L. Russell4, \\ Carolina Correa-Ospina ${ }^{4}$, Bentotage S. M. Fernando ${ }^{3}$, Shafagh A. Waters ${ }^{5}$, Natalia Castaño-Rodríguez ${ }^{1}$, \\ Si Ming Man ${ }^{6}$, Nicodemus Tedla ${ }^{3}$, Marc R. Wilkins ${ }^{1,4}$ and Nadeem O. Kaakoush ${ }^{3^{*}}$ (D)
}

\begin{abstract}
Background: The enrichment of Gram-negative bacteria of oral origin in the esophageal microbiome has been associated with the development of metaplasia. However, to date, no study has comprehensively assessed the relationships between the esophageal microbiome and the host.

Methods: Here, we examine the esophageal microenvironment in gastro-esophageal reflux disease and metaplasia using multi-omics strategies targeting the microbiome and host transcriptome, followed by targeted culture, comparative genomics, and host-microbial interaction studies of bacterial signatures of interest.

Results: Profiling of the host transcriptome from esophageal mucosal biopsies revealed profound changes during metaplasia. Importantly, five biomarkers showed consistent longitudinal changes with disease progression from reflux disease to metaplasia. We showed for the first time that the esophageal microbiome is distinct from the salivary microbiome and the enrichment of Campylobacter species as a consistent signature in disease across two independent cohorts. Shape fitting and matrix correlation identified associations between the microbiome and host transcriptome profiles, with a novel co-exclusion relationship found between Campylobacter and napsin B aspartic peptidase. Targeted culture of Campylobacter species from the same cohort revealed a subset of isolates to have a higher capacity to survive within primary human macrophages. Comparative genomic analyses showed these isolates could be differentiated by specific genomic features, one of which was validated to be associated with intracellular fitness. Screening for these Campylobacter strain-specific signatures in shotgun metagenomics data from another cohort showed an increase in prevalence with disease progression. Comparative transcriptomic analyses of primary esophageal epithelial cells exposed to the Campylobacter isolates revealed expression changes within those infected with strains with high intracellular fitness that could explain the increased likelihood of disease progression.
\end{abstract}

Conclusions: We provide a comprehensive assessment of the esophageal microenvironment, identifying bacterial strain-specific signatures with high relevance to progression of metaplasia.

Keywords: Esophagus, Metaplasia, Adenocarcinoma, Microbiome, Transcriptome, Campylobacter, intracellular survival

\footnotetext{
* Correspondence: n.kaakoush@unsw.edu.au

${ }^{3}$ School of Medical Sciences, Faculty of Medicine, UNSW Sydney, Sydney, NSW 2052, Australia

Full list of author information is available at the end of the article
}

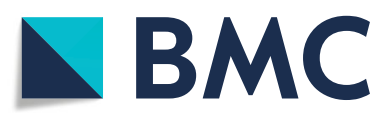

(- The Author(s). 2021 Open Access This article is licensed under a Creative Commons Attribution 4.0 International License, which permits use, sharing, adaptation, distribution and reproduction in any medium or format, as long as you give appropriate credit to the original author(s) and the source, provide a link to the Creative Commons licence, and indicate if changes were made. The images or other third party material in this article are included in the article's Creative Commons licence, unless indicated otherwise in a credit line to the material. If material is not included in the article's Creative Commons licence and your intended use is not permitted by statutory regulation or exceeds the permitted use, you will need to obtain permission directly from the copyright holder. To view a copy of this licence, visit http://creativecommons.org/licenses/by/4.0/. The Creative Commons Public Domain Dedication waiver (http://creativecommons.org/publicdomain/zero/1.0/) applies to the data made available in this article, unless otherwise stated in a credit line to the data. 


\section{Background}

Esophageal cancer is the 7th most common cancer and the 6th most common cause of cancer-related mortality [1]. There are two major subtypes of esophageal cancer, squamous cell carcinoma (ESCC) and adenocarcinoma (EAC), with levels of the former decreasing while those of the latter increasing globally $[1,2]$. Survival of patients appears to be improving with time; however, 5-year survival rates remain very poor due to late diagnosis $[1,2]$.

There are well-established risk factors for the development of EAC, including reflux symptoms, obesity, sex, age, and ethnicity [3]. The chronic exposure to refluxate of acid and bile leading to gastro-esophageal reflux disease (GERD) is the strongest known risk factor [3]. Long-term GERD leads to metaplastic changes, termed Barrett's esophagus (BAR), a known precursor of EAC [4]. The burden of GERD is also increasing globally [5], contributing to the increases in BAR incidence, and consequently EAC.

The esophageal microbiota has been proposed as a contributor to the progression towards BAR $[6,7]$; however, the evidence remains relatively scarce, inconsistent $[8,9]$, and more supportive of a role for specific pathobionts rather than global composition shifts [10, 11]. The limited number of studies investigating the human esophageal microbiome has by large focused on changes in prevalence or relative abundances of microbial taxa, with only one study by our group looking at changes in microbial function [10]. While these studies have provided insights into possible microbial agents of esophageal disease, they have lacked validation and have not causally linked the microbial changes with changes in the local host response, and there have not been attempts at delineating disease-promoting capabilities of microbial signatures of interest.

Given the similarities between the etiology of EAC and gastric adenocarcinoma [11], what is anticipated is that if microbial species are involved in disease progression then they are likely involved in the early stages of the pathological cascade (i.e., GERD and metaplasia). Multiomics strategies and integrative analyses have been utilized effectively to identify factors central to disease etiology [12]. Thus, we employed a multi-omics strategy to profile the esophageal microenvironment in GERD and BAR, identifying host and microbial signatures putatively associated with disease progression in the esophagus. The associated bacterial species were then isolated from the same patients through targeted culture strategies, and novel genomic features associated with increased intracellular fitness in primary immune cells were identified. Screening of these microbial genomic features in shotgun metagenomics data of another cohort revealed a differential prevalence across the early stages of the EAC cascade. Co-culture of bacterial isolates with primary esophageal epithelial cells identified transcriptomic changes unique to those of relevance to disease.

\section{Methods \\ Recruitment of subjects and nucleic acid extraction}

To assess the esophageal microenvironment in GERD and the development of metaplasia, 48 subjects who underwent upper gastrointestinal endoscopy at the Prince of Wales Hospital (Sydney) for examination of their gastrointestinal symptoms were recruited prospectively (Table 1). Subjects who had a normal esophagus by histological assessment were considered controls. Subjects who had been prescribed antibiotics or non-steroidal antiinflammatory drugs in the 2-month period prior to recruitment as well as subjects with other esophageal disease (squamous cell carcinoma, $n=1$ ) were excluded. Two research samples, an esophageal mucosal biopsy and a saliva sample, were collected at endoscopy in addition to the required clinical samples. Researchers were blinded to the results of the histological analysis until sequencing was completed. Patients with GERD $(n=13)$ were uniformly graded by one endoscopist according to the Los Angeles

Table 1 Clinical diagnosis, symptoms, and information of subjects. Within the new prospective cohort, patients with tissue metaplasia (MET) were older than patients with normal esophagi ( $P=0.032$-ANOVA post hoc Tukey's test). While mean BMl increased with the progression of the cascade, no significant differences were observed (ANOVA post hoc Tukey's test). As the researchers were blinded to clinical diagnosis until after sequencing, one patient found to have squamous cell carcinoma was excluded from further analyses. Within the published cohort, no significant differences in age were identified across groups $\left(F_{2,97}=\right.$ 1.19, $P=0.31$; ANOVA). Patient BMI was not available. NORM normal, GERD gastro-esophageal reflux disease, MET metaplasia, $M$ male, $Y$ yes, PPI proton pump inhibitor, BMI body mass index. Age and BMI \pm standard error of the mean

\begin{tabular}{llllllll}
\hline Cohort & Disease & Number (\%) & Age (years) & Gender (M) & BMI & Reflux symptoms (Y) & PPI (Y) \\
\hline Prospective & NORM & $27(57.4)$ & $51.2 \pm 3.1$ & 7 & $25.4 \pm 0.7$ & 8 & 13 \\
& GERD & $13(27.6)$ & $57.3 \pm 4.9$ & 5 & $26.3 \pm 1.2$ & 6 & 6 \\
& MET & $7(14.9)$ & $68.8 \pm 4.0$ & 4 & $28.6 \pm 1.5$ & 2 & 4 \\
Published & NORM & $59(59.0)$ & $53.1 \pm 1.9$ & 21 & - & 1 & 32 \\
& GERD & $29(29.0)$ & $52.0 \pm 2.5$ & 8 & - & 28 & 9 \\
& MET & $12(12.0)$ & $59.2 \pm 3.6$ & 10 & - & 9 & 9 \\
\hline
\end{tabular}


classification [13] and were classified into the grades A ( $n$ $=9)$ and B $(n=4)$. No other findings at endoscopy were reported in any of the patients recruited. Nucleic acids were extracted from saliva and esophageal mucosal biopsies using the AllPrep DNA/RNA Mini Kit (Qiagen). RNA was further purified using $8 \mathrm{M} \mathrm{LiCl}$. In addition to the current prospectively recruited cohort, $16 \mathrm{~S}$ rRNA amplicon and shotgun metagenomics sequencing data from a previously published cohort (Table 1) [10], recruited at the Prince of Wales Hospital (Sydney), were employed. Data from esophageal brushings of 100 subjects [normal (NORM), $n$ = 59; GERD, $n=29$; metaplasia (MET), $n=12]$ who underwent upper gastrointestinal endoscopy for examination of their gastrointestinal symptoms were assessed.

\section{Host transcriptomics}

To investigate the host transcriptome profile during progression to metaplasia, RNA was prepared for sequencing using the SMARTer Stranded Total RNA Pico preparation kit and sequenced using NovaSeq 6000 chemistry (S2 100bp paired-end run). The tool Salmon which uses a quasi-mapping approach was employed for quantifying transcript abundance from RNA-seq reads [14]. Cell type analysis was performed using CIBERSORTx [15]. Multivariate statistics were applied as described above for the microbiota data. The $\mathrm{R}$ package DESeq2 was used to identify differentially expressed genes across specific comparisons [16]. Pathway and disease analyses were performed using Enrichr [17].

Three tools were used for identification of all major types of alternative splicing events (Exon skipping, intron retention, A5SS, A3SS, and mutually exclusive exons). These included rMATS [18], Whippet [19], and PSI-Sigma [20]. In-house scripts [21] were developed in python to identify splicing events which led to possible functional switches. These included biotype changes (e.g., from transcripts coding for functional proteins in one condition to the transcripts leading to proteins marked for nonsense-mediated decay or processed transcripts without a protein product in the other condition) or the events which lead to changes in protein product due to frame-shift, thus, resulting in complete or partial loss of functional domains. The Bioconductor/R packages maser [22] and drawProteins [23] were employed for visualizations of the alterative splicing events in transcripts in context of their protein products. PANTHER was employed for gene ontology enrichment analysis [24]. The tool CIRCexplorer2 [25] was used for identification and characterization of circular RNAs. Fusion genes were identified using the tool STAR-Fusion [26]. This tool uses the STAR aligner to map Illumina short reads, and then proceeds to use the junction as well as the spanning reads from the mapping output to the reference annotation to identify gene fusions.

\section{S rRNA amplicon sequencing}

To examine the differences between the salivary (S) and esophageal (E) microbiotas, as well as determine any changes with disease progression, the full-length $16 \mathrm{~S}$ rRNA gene was amplified using a KAPA HiFi HotStart PCR Kit and the primers 27F (/5AmMC6/ gcagtcgaacatgtagctgactcaggtcac AGRGTTYGATYMTGGCTCAG) and 1492R (/5AmMC6/ tggatcacttgtgcaagcatcacatcgtag RGYTACCTTGTTACGACTT) and sequenced on a PacBio RSII platform. The cycling conditions were $95^{\circ} \mathrm{C}$ for $3 \mathrm{~min}$, followed by 34 cycles for biopsies or 27 cycles for saliva, of $95^{\circ} \mathrm{C}$ for $30 \mathrm{~s}, 57^{\circ} \mathrm{C}$ for $30 \mathrm{~s}$, and $72^{\circ} \mathrm{C}$ for $60 \mathrm{~s}$. Raw reads were analyzed using Mothur v1.39.1 [27, 28]. The resultant operational taxonomic unit (OTU) count matrix was used for statistical analysis (mean read depth: $1893 \pm 101$ clean reads/sample). OTUs generated from this data were termed pOTUs.

The V4 region of the 16S rRNA gene was also amplified using the earth microbiome primers (515F-806R), and sequencing was performed with Illumina MiSeq $2 \times$ $250 \mathrm{bp}$ chemistry at the Ramaciotti Centre for Genomics, UNSW Sydney, as previously described [10]. Raw reads were analyzed using Mothur v1.44.0 and vsearch v2.13.3 [27, 28]. E24 did not sequence to saturation and was removed along with its matching saliva sample (S24) from downstream analyses. The subsampled OTU count matrix was used for analysis (read depth: 13898 clean reads/sample). OTUs generated from this analysis were termed iOTUs. The prospective and published cohorts (Table 1) were then combined, and raw reads again analyzed using Mothur v1.44.0 and vsearch v2.13.3. The subsampled OTU count matrix was used for analysis (read depth: 13,898 clean reads/sample). OTUs generated from this analysis were termed ciOTUs.

$\alpha$-diversity measures, Euclidean distances, Bray-Curtis resemblances, principal coordinate analysis (PCoA), distance-based redundancy analysis (dbRDA), ANOSIM, and distance-based linear models (permutational multivariate ANOVA) were calculated using Primer-E v6 (Quest Research Limited; Auckland, New Zealand). The models included the variables: location (saliva or esophageal), subject age, sex, proton pump inhibitor (PPI) use, body mass index (BMI), reflux symptoms, and disease (NORM, GERD, MET) and were tested against Euclidean distances for $\alpha$-diversity measures and Bray-Curtis resemblances for beta-diversity. Per taxon analyses were performed using LEfSe [29]. Source tracking of esophageal taxa against saliva samples was performed using SourceTracker [30], within the Metagenomics for Environmental Microbiology Galaxy framework [31]. All other tests were performed using GraphPad Prism v8 (GraphPad Software; San Diego, CA, USA). 


\section{Associations between microbiome and host transcriptome}

To determine if any associations exist between the host transcriptome and microbiota profiles, correlations of resemblance matrices were performed using the RELATE function in Primer-E v6. Procrustes and protest analyses were performed using the R package "vegan." Tests were performed on both PCoA and dbRDA axes calculated for Bray-Curtis resemblance matrices of microbiome (transformed relative abundances) and host transcriptome data (normalized counts). Non-parametric correlations were calculated using the framework outlined in Reshef et al. [32] and accessible through the $\mathrm{R}$ package "minerva." Inputs were transformed relative abundances of Campylobacter taxa and normalized transcript counts.

\section{Isolation of motile bacterial species from the patient cohort}

To culture members of the microbiota of relevance to disease progression, patients' saliva and esophageal mucosal biopsy samples were resuspended in $1 \times$ phosphate buffered saline (PBS), homogenized, and cultured at $37^{\circ} \mathrm{C}$ for $48 \mathrm{~h}$ on Horse Blood Agar (HBA) [Blood Agar Base No. 2 (Oxoid, Melbourne, Vic, AU) with 6\% defibrinated horse blood (Oxoid)] containing $10 \mu \mathrm{g} / \mathrm{ml}$ vancomycin (Sigma-Aldrich; Sydney, NSW, AU) under anaerobic conditions with hydrogen enrichment $[10 \%$ hydrogen, $5 \% \mathrm{CO}_{2}, \sim 0.5 \% \mathrm{O}_{2}$ ]. These conditions were generated using an AnaeroGen gas pack (Oxoid) and $0.073 \mathrm{~g}$ sodium borohydride (Sigma-Aldrich). The resultant culture was filtered through a $0.6 \mu \mathrm{M}$ membrane (Millipore, Melbourne, Vic, AU), and the filtrate subcultured on HBA under the same conditions. Single colonies were selected from each culture's filtrate and were harvested into Brain Heart Infusion broth and glycerol $(70: 30 \mathrm{v} / \mathrm{v})$. To confirm isolates were Campylobacter species, the $16 \mathrm{~S}$ ribosomal RNA gene was amplified directly from colonies through Campylobacter-specific PCR using the $\mathrm{C} 412 \mathrm{~F}$ and C1288R primer pair (40 cycles of $95^{\circ} \mathrm{C}$ for $10 \mathrm{~s}, 55^{\circ} \mathrm{C}$ for $10 \mathrm{~s}$, and $72{ }^{\circ} \mathrm{C}$ for $45 \mathrm{~s}$ ). For additional validation, PCR products were Sanger sequenced using BigDye chemistry and their identity confirmed using BLASTn searches against the NCBI database. Positive isolates were stored at $-80^{\circ} \mathrm{C}$ for further use.

\section{Culture of primary macrophages and infection with bacterial isolates}

A range of Campylobacter species are known to invade and survive within epithelial cells; however, there is no consistent association between their capacity to do so and the disease status of the host from which they were isolated from. We postulated that isolates that induce more amplified responses from immune cells or that could overcome their defenses would more likely correspond to persistent pathobionts that have a chronic effect on the host.

Primary human monocyte-derived macrophages were prepared from buffy coats $(100 \mathrm{ml})$ obtained from eight healthy donors through the Australian Blood Services (Australian Red Cross material supply agreement: 1801NSW-06) under strict LPS-minimized conditions as described [33]. In brief, PBMCs were isolated using density gradient centrifugation (Ficoll-Paque Plus; Amersham Biosciences). PBMCs washed twice with PBS were suspended at $\sim 1 \times 107$ cells $/ \mathrm{ml}$ in RPMI 1640 containing $2 \mathrm{mM}$ L-glutamine, $10 \mathrm{U} / \mathrm{ml}$ penicillin and 100 $\mathrm{mg} / \mathrm{ml}$ streptomycin, and $10 \%$ heat-inactivated $\mathrm{Ab}$ serum (Sigma). Cells were incubated at $37^{\circ} \mathrm{C}$ and $5 \%$ $\mathrm{CO}_{2}$ in 24-well Corning Costar plates and non-adherent cells removed after $1.5-2 \mathrm{~h}$ yielding $2-3 \times 10^{6}$ monocytes $/ \mathrm{ml} /$ well (>90\% as confirmed by CD14 staining). Cells were then cultured in RPMI 1640 complete media containing 10\% AB serum supplemented with $20 \mathrm{ng} / \mathrm{ml}$ M-CSF (BioSource) for 3 days, then washed twice with PBS and cultured for another 4 days in culture medium without M-CSF. Primary macrophages were infected with each of the different Campylobacter isolates from our cohort at a MOI of 100, supernatants were collected ( 4 and $18 \mathrm{~h}$ ) for multiplex ELISA, and gentamicin protection assays were performed as previously described [34]. Then, $1 \mu \mathrm{M}$ Latrunculin A (Sigma) was added to a subset of experiments to block phagocytosis. The acidification of lysosomes upon infection was tracked using 2.5\% LysoTracker Green DND-26 (Thermo Fisher; North Ryde, NSW, AU) as outlined by the manufacturer.

\section{Multiplex ELISA}

To establish the inflammatory response of healthy primary macrophages to patient Campylobacter isolates, 34 cytokines and chemokines were measured in the coculture supernatants using the Cytokine \& Chemokine 34-Plex Human ProcartaPlex ${ }^{\mathrm{Tm}}$ Panel 1A (Jomar Life Research; Scoresby, VIC, Australia) according to the manufacturer's instructions. A Luminex MAGPIX instrument with $x P O N E N T$ software (Luminex Corporation; Northbrook, IL, USA) was calibrated with MAGPIX Calibration and Performance Verification Kits (EMD Millipore; Billerica, MA, USA) and employed to acquire data. Data was analyzed with Multiplex Analyst software version 5.1 (Luminex) as the Median Fluorescent Intensity using spline curve-fitting for calculating analyte concentrations.

\section{Genome sequencing of isolates and comparative genomics}

To identify genetic features that differentiate the Campylobacter isolates, bacteria were grown as described and DNA was extracted using the Isolate II 
Genomic DNA Kit (Bioline; Cat number: BIO-52066). DNA from each isolate was prepared for sequencing on individual PacBio RSII sequencing cells using the 10-20 kb Genomic RSII library preparation kit. Bacterial DNA was also prepared using Nextera XT DNA library preparation kit and sequenced using MiSeq v3 $2 \times 300$ bp chemistry at the Ramaciotti Centre for Genomics. The package SolexaQA [35] was used to calculate sequence quality statistics and trim the input fastq files by quality. The analysis included quality trimming of the reads to the user-supplied quality cutoffs using the module dynamictrim. This was followed by trimming the reads by user-defined length cutoff using the module lengthsort. The assembly of individual bacterial strains was performed using the Canu assembler [36], specialized for noisy single-molecule sequences. The tool Pilon [37] was then employed to refine the draft assembly by correcting bases, fixing mis-assemblies, and filling gaps with the help of high accuracy Illumina short-read datasets from the same samples. The final refined genome assemblies were annotated using the highly accurate and fast command line tool Prokka [38]. Average nucleotide identity (ANI) was calculated using OrthoANI [39] and genomes were visualized using GView [40]. Comparative genomics to identify strain-specific orthologous proteins was performed using the tool Proteinortho [41].

\section{Assessment of resistance to lysozyme}

The susceptibility of isolates to lysozyme was assessed either by exposure to $1 \mathrm{mg} / \mathrm{ml}$ human lysozyme (Sigma) or a combination of $0.3 \mathrm{mg} / \mathrm{ml}$ lysozyme and $3 \mathrm{mg} / \mathrm{ml}$ human lactoferrin (Sigma) for $6 \mathrm{~h}$.

\section{Generation of bacterial mutants deficient in Lprl_01601 and Lprl_00928}

The C. concisus $\Delta$ LprI_01601 and C. rectus $\Delta$ LprI_00928 mutants were generated using an adapted protocol involving allelic exchange [42]. A linear DNA template was designed to contain fragments of about $500 \mathrm{bp}$ upstream and downstream of the target gene flanking a kanamycin resistance cassette from Campylobacter jejuni (accession number: M29953.1). Both linear templates, incorporated into a pET-3a plasmid within the BamHI site, were generated by GenScript (Piscataway, NJ, USA). The C. concisus template was amplified from the plasmid by flanking primers (CC1601A: ACGAGG CTAGCGTTTTTAGC; CC1601D: GCTAATAGTTTT CAAGCTGCATTC), purified, methylated using $200 \mu \mathrm{g}$ of C. concisus ESOS44-1 lysates with 0.4 mM S-Adenosylmethionine (Sigma) as previously outlined [43], and naturally transformed into $C$. concisus ESOS44-1 grown for $4 \mathrm{~h}$. The $C$. rectus template was amplified from the plasmid by flanking primers (CR00928F1: TCCACAGA AAAGCTCATATCC; CR00928R1: TTCGTACTCGCA
GCTCGTCAA), purified, methylated using $200 \mu \mathrm{g}$ of $C$. rectus ESOS44-4 lysates as above, and naturally transformed into $C$. rectus ESOS44-4 grown for $4 \mathrm{~h}$. Isolates were transferred onto kanamycin-containing HBA plates $(20 \mu \mathrm{g} / \mathrm{ml})$ after $18 \mathrm{~h}$. The C. concisus mutant was validated through PCR of the target gene using various combinations of the flanking primers with primers internal to LprI_01601 (1601F: GTAAGCAATACACAAG ATATTGAAG; 1601R: TTGCTAACTCTTCCGCTCTT ). The $C$. rectus mutant was validated using amplification with the above primers (> $1700 \mathrm{bp}$ fragment) and Sanger sequencing with BigDye Terminator v3.1 chemistry. Of note, transformation efficiency of $C$. rectus was lower as the bacterium had a higher tolerance to kanamycin, and thus, an increased concentration for selection may be more suitable.

\section{Expression of recombinant Lprl_01601 and immunoprecipitation}

The sequence of LprI_01601 from ESOS44-1 with a Cterminal His-tag was inserted into a pET-30a $(+)$ plasmid between NdeI and HindIII, expressed in E. coli, and purified by Ni column. Plasmid sequences were validated by Sanger sequencing, and protein expression purification was validated by SDS-PAGE and anti-His-Tag Western blot analysis. All services were performed by GenScript. Interaction partners were then identified by using LprI 01601 as a bait and primary macrophage non-detergent cell lysates as target co-immunoprecipitated with magnetic bead conjugated anti-His-tag monoclonal antibody as previously described [44]. The experiment was repeated twice, and all fractions were analyzed using LC/MS-MS [45]. Only proteins identified in both repeats with a mascot score $>60$ in at least one experiment and not identified in either of the negative controls (anti-His-tag IP without LprI_01601) were retained. The list was then further filtered for keratins, immunoglobulins, histones, and ribosomal proteins.

\section{Co-culture of primary esophageal epithelial cells with Campylobacter isolates}

Human primary esophageal epithelial cells (Cell Biologics; H-6046) were grown on the recommended Complete Human Epithelial Cell Medium (Cell Biologics; H-6621) in coated 24-well tissue culture plates at a concentration of $1.5 \times 10^{4}$ cells $/ \mathrm{ml}$. The cell culture media was replaced with antibiotic-free media then representative Campylobacter isolates (ESOS13-1, ESOS18-1, ESOS14-1, ESOS331, ESOS44-1, and ESOS44-4) grown as described above, were added to the human cells at a MOI of 10 for $4 \mathrm{~h}$. RNA was extracted using the RNeasy Plus Mini Kit (Qiagen) and prepared using the Stranded mRNA library kit (Illumina). Libraries were then sequenced on the NovaSeq 6000 system (flow cell type: SP; chemistry: $1 \times 100$ bp) 
producing a mean 21,735,733 $\pm 311,229$ clean reads across all samples. Data were analyzed as above, utilizing Salmon [14] and DESeq2 [16] to identify differentially expressed genes, as well as Enrichr [17] for pathway analyses.

\section{Results}

Profound changes in the host esophageal transcriptome occur in metaplasia but not GERD

To examine the changes in the host transcriptional profile in GERD and MET, bulk shotgun sequencing of RNA from biopsy samples from prospectively recruited subjects (Table 1) was performed. The immune cell profile within the biopsies was estimated using CIBERSORTx, and analyzed against all variables (age, sex, PPI use, BMI, reflux symptoms, and disease) with a distancebased linear model. No significant differences were identified in the cell profiles. However, a borderline nonsignificant difference was found for disease (Pseudo-F: 2.0, $P=0.057, \mathrm{df}=41)$ that reached significance on oneway analysis against disease ( $R=0.152, P=0.035$; ANOSIM) and was driven by differences between MET and the other groups (NORM: $R=0.291, P=0.018$; GERD: $R=0.214, P=0.023)$. Specifically, lower levels of resting mast cells and higher levels of active mast cells were found in MET samples when compared to NORM and GERD (Fig. 1A, B).

Changes in host transcription profiles were then assessed using a distance-based linear model on logtransformed counts (Bray-Curtis resemblance) accounting for same variables as above, with the strongest differences observed for disease (Pseudo-F: 11.6, $P=$ 0.001, $\mathrm{df}=41$ ), followed by BMI (Pseudo-F: $3.1, P=0.012$, $\mathrm{df}=41$ ), age (Pseudo-F: $2.8, P=0.016, \mathrm{df}=41$ ), and sex (Pseudo-F: 2.1, $P=0.033, \mathrm{df}=41$ ). The changes in transcript levels in disease were driven once again by differences between MET samples and the other groups (NORM: $t=4.05, P=0.001, \mathrm{df}=29$; GERD: $t=2.94, P=$ 0.001 , $\mathrm{df}=17$, PERMANOVA), with no difference observed when NORM and GERD were compared $(t=1.12$, $P=0.14, \mathrm{df}=34$, PERMANOVA). Differentially expressed transcripts were then identified, with only 12 significantly regulated between NORM and GERD (Fig. 1C; Additional file 1: Table S1) as compared to 7884 between NORM and MET (Fig. 1D, Additional file 1: Table S2), confirming the multivariate analyses. Notably, of the 12 differentially expressed transcripts in GERD, 5 were differentially expressed in the same direction in MET (Fig. 1E), showing some consistency across the two stages.

Pathway analysis of differentially regulated transcripts in MET was then performed, with key pathways known to be altered in BAR identified as enriched. These pathways included mucin glycan biosynthesis, oxytocin signaling, pathways related to carcinogenesis, and those related to acid and bile secretion (Fig. 1F; Additional file
1: Table S3). Enrichment of Fc gamma R-mediated phagocytosis and Lysosome-related proteins was also observed (Additional file 1: Table S3). Tissue type and disease enrichment analyses confirmed expression in MET resembled that in the colon, and specifically, colonic malignancy (Fig. 1G). FOXA2, Cathepsin E (CTSE), REG4, TFF1, TFF2, the bicarbonate channel CFTR, and PDZdomain-containing mucins (MUC17, MUC3A, and MUC12) were among the most highly upregulated transcripts in MET.

In addition to cell types and expression profiles, changes in splicing, circular RNAs, and fusion events were also interrogated. A substantial number of putative splicing events were identified in both GERD and MET when compared to NORM samples (Additional file 1: Table S4), with a strict assessment of exon skipping and intron retention events highlighting that a number of these events can potentially lead to changes in protein amino acid sequence due to nonsense-mediated decay (Additional file 2: Figures S1, S2; Additional file 1: Table S5). Enrichment analysis showed that differentially spliced transcripts in GERD were populated by transcripts involved in the cellular response to stress (fold enrichment $=3.37, P=4.25 \times 10^{-9}, \mathrm{FDR}=6.75 \times 10^{-5}$ ), whereas those in MET were populated by transcripts involved in stress granule formation (fold enrichment = 29.79, $P=1.75 \times 10^{-6}, \mathrm{FDR}=0.0278$ ).

Differences in the prevalence of circular RNAs (Additional file 2: Figure S3A; Additional file 1: Table S6) and fusion events (Additional file 2: Figure S3B; Additional file 1: Table S7) across the three groups were also identified, in the majority of cases MET being different to NORM and GERD. Two notable exceptions were the circular RNAs CUL6, showing consistent prevalence in GERD and MET, as well as GOLM1 showing a stepwise increase in disease (Additional file 2: Figure S3A).

The above findings indicate that while some changes in host profile can be observed in GERD, most changes occur at the stage of or during progression to MET (Fig. 1H).

\section{The esophageal microbiota is distinct from the salivary microbiota with Campylobacter being enriched in reflux and metaplasia}

To establish that the esophageal microbiota is a distinct microbial community, matched saliva and esophageal samples from the same recruited subjects (Table 1) were first profiled using full-length 16S RNA amplicon sequencing (PacBio) and community metrics were compared. The esophageal microbiota had significantly lower alpha diversity measures (Fig. 2A) and significantly different composition (Fig. 2B), even after correction for subject age, sex, PPI use, BMI, reflux symptoms, and 
A

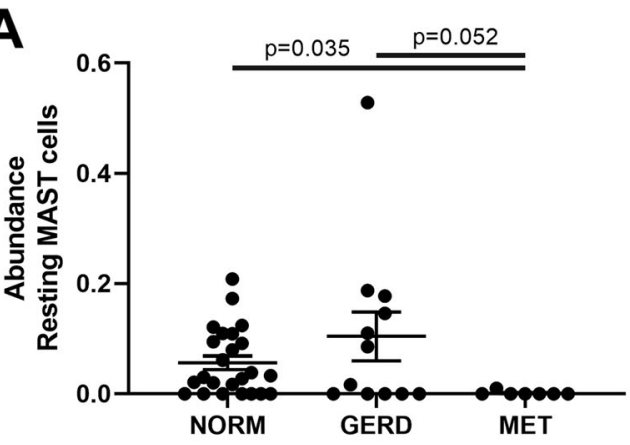

C

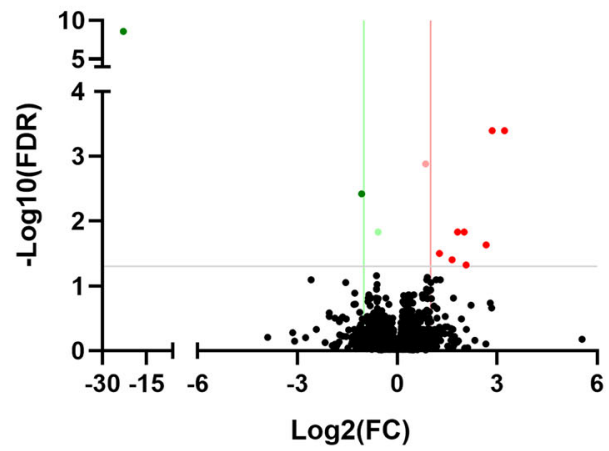

E

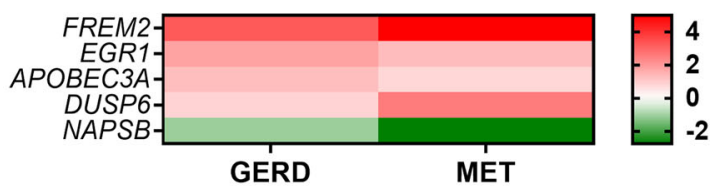

G
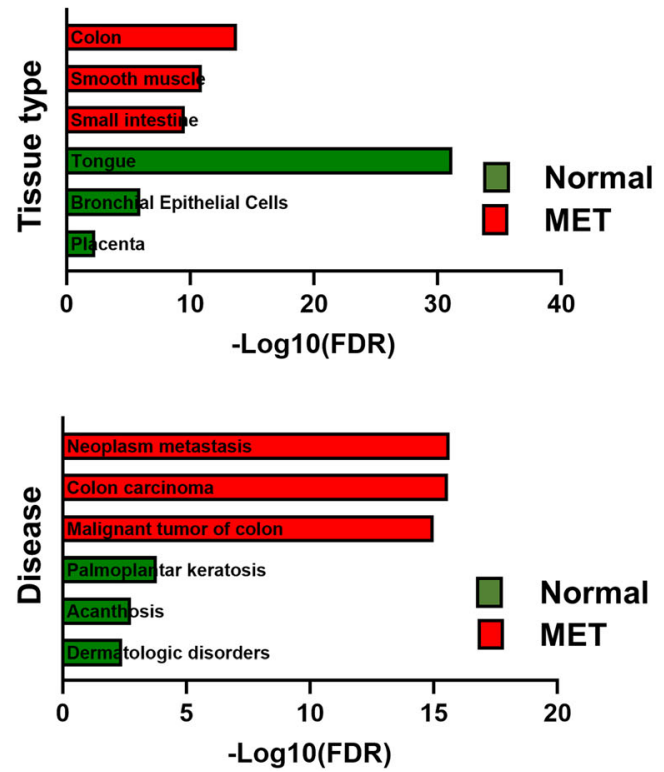

B

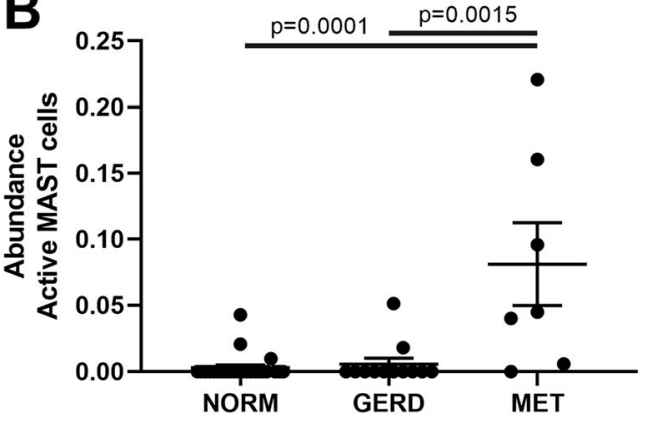

D

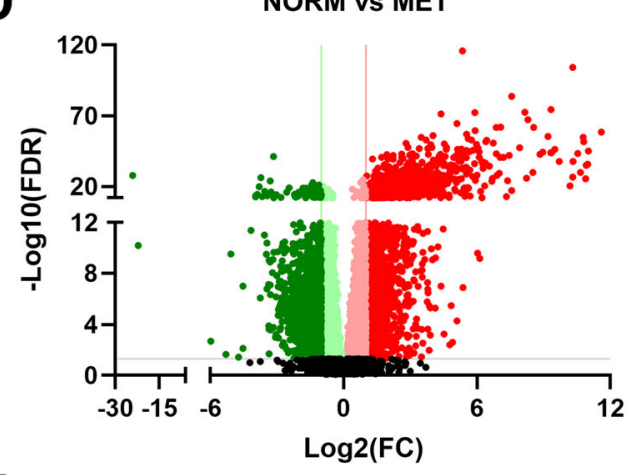

$\mathbf{F}$
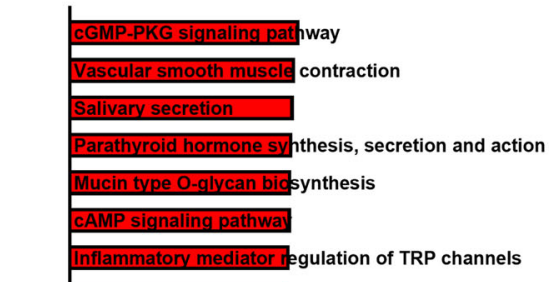

$\sum_{\substack{\pi \\ \frac{1}{3}}}^{\infty}$ Other glycan degradation

Glycerophospholipid m:tabolism

Gastric acid secretion

Oxytocin signaling patt way
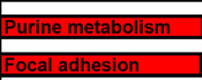

Circadian entrainment

Chemical carcinogentsis

Normal

Pancreatic secretion

Basal cell carcinoma

Hippo signaling pathway

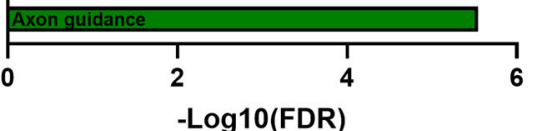

H

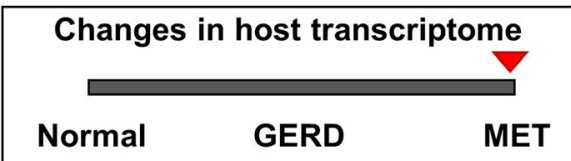

Fig. 1 (See legend on next page.) 
(See figure on previous page.)

Fig. 1 Host transcriptome changes in the esophagus of patients with GERD and metaplasia. Shotgun sequencing of ribosomal-depleted RNA was performed on a NovaSeq platform. A Changes in predicted abundance of resting mast cells across the different groups. B Changes in predicted abundance of active mast cells across the different groups. Abundances were calculated using CIBERSORTx and statistical differences were tested using Kruskal-Wallis with Dunn's multiple comparisons test. C Volcano plot of differentially expressed genes between NORM and GERD samples. D Volcano plot of differentially expressed genes between NORM and MET samples. Differential expression was calculated using DESeq2. Red, upregulated in disease state, Green, downregulated in disease state. E Genes consistently regulated across GERD and MET when compared to NORM samples. Scale bar is fold-change. F Pathway analysis of differentially expressed genes in MET samples. G Tissue type and disease analyses of differentially expressed genes in MET samples. All analyses were conducted using Enrichr. $\mathbf{H}$ Significant changes in the host esophageal transcriptome occur at the time of metaplasia

disease. Many taxa were significantly different in relative abundance across sample types (Additional file 1: Table S8), the taxon showing the largest differential relative abundance being Streptococcus pOTU1 (LDA $=5.16, P<$ 0.0001; Fig. 2C). Despite the differences between communities, as expected, source tracking of microbial counts indicated that most of the esophageal taxa originated from the oral cavity (mean \pm STD: $0.84 \pm 0.27$; 95\% CI 0.76-0.92).

Given that the two communities were distinct, their associations with different predictors were analyzed independently. In a model that included subject age, sex, PPI use, BMI, reflux symptoms, and disease, a significant association was identified between disease and esophageal species richness (Pseudo-F: 7.4, $P=0.005, \mathrm{df}=45$ ), marked by an increase in richness with disease progression (Fig. 2D). Models incorporating the same variables also identified a significant shift in esophageal microbiota composition (Pseudo-F: $1.4, P=0.02, \mathrm{df}=45$; Fig. $2 \mathrm{E}$ ), which was confirmed on one-way analysis $(R=0.178, P=0.008$; ANOSIM). To identify the taxa responsible for this shift, per taxon analyses were performed using LEfSe (Additional file 1: Tables S9-11), and a striking enrichment of Campylobacter was observed in disease (Fig. 2F,G; GERD: LDA $=3.53, P=0.018$; DIS: LDA $=3.66, P=0.012$ ). No associations were observed with the saliva microbiota, except for a trend towards a shift in composition with BMI (Pseudo-F: $1.1, P=0.063, \mathrm{df}=45)$.

To confirm the observed outcomes using a different platform, the V4 region of the $16 \mathrm{~S}$ rRNA gene was sequenced (Illumina), and the same tests applied. Differences were once again observed between the saliva and esophageal communities, with lower alpha diversity measures in the esophagus (Additional file 2: Figure S4A), a compositional shift (Additional file 2: Figure S4B), and higher relative abundance of Streptococcus iOTU2 (LDA $=4.98, P<0.0001$; Additional file 2: Figure S4C; Additional file 1: Table S8). Consistently, a trend towards increased esophageal species richness in disease was observed (Pseudo-F: 4.3, $P=0.052, \mathrm{df}=44$; Additional file 2: Figure $\mathrm{S} 4 \mathrm{D}$ ), in addition to an association between esophageal species richness with BMI (Pseudo-F: 3.5, $P$ $=0.048, \mathrm{df}=44)$. Differences in esophageal microbiota composition with disease had a similar effect size to above but did not reach significance (Pseudo-F: 1.37, $P=$ 0.12 , df $=44)$. Notably however, when patients with GERD and Barrett's were combined, the associations between richness and disease (Pseudo-F: 6.3, $P=0.018, \mathrm{df}$ $=44$ ) as well as composition and disease (Pseudo-F: 1.6, $P=0.038, \mathrm{df}=44$ ) reached significance, suggesting that the resolution from profiling a smaller fragment may contribute to the statistical power to identify differences. The association between the saliva microbiota composition and BMI was confirmed using this platform (Pseudo-F: $2.1, P=0.017, \mathrm{df}=44$ ). To validate the taxa driving these differences, LEfSe analysis were performed (Additional file 1: Tables S9-11), and a taxon classified to Campylobacter was found to be enriched in disease (GERD: LDA $=3.28, P=0.0021$; DIS: LDA $=3.17, P=$ 0.0029; Additional file 2: Figure S4E,F). This taxon (iOTU36) was also more prevalent in disease [NORM: 26.9\% (7/26); GERD: 76.9\% (10/13); MET: $57.1 \%$ (4/7); $\left.\chi^{2}=9.17, P=0.010\right]$.

To validate the relevance of Campylobacter in a larger cohort, the current cohort was re-analyzed in combination with a previously published cohort of esophageal brushings from subjects with normal esophagi, GERD, or metaplasia [Table 1 [10];]. While a strong batch effect was detected (Additional file 2: Figure S5A), the same Campylobacter taxon (cOTU65; different OTU assignment but same consensus sequence) was consistently found to be enriched in disease (GERD: LDA $=3.04, P=$ 0.0018; MET: $\mathrm{LDA}=2.87, P=0.021$; DIS: $\mathrm{LDA}=3.02$, $P=0.00048$; Additional file 2: Figure S5B,C; Additional file 1: Tables S9-11).

The findings indicate that the esophageal microbiota is distinct, and changes in richness and composition occur at GERD (Fig. $2 \mathrm{H}$ ) and are driven in part by consistent enrichment of specific taxa such as Campylobacter.

\section{Campylobacter levels were correlated with inferred levels of active mast cells and expression of a lysosomal transcript}

The relationship between the esophageal microbiome and host transcriptome in this cohort was assessed to determine if any associations exist. Significant concordance between the microbiome (PacBio) and transcriptome data sets (PCoA: $\mathrm{m}_{12}=0.454, P=0.001$; dbRDA: 
A
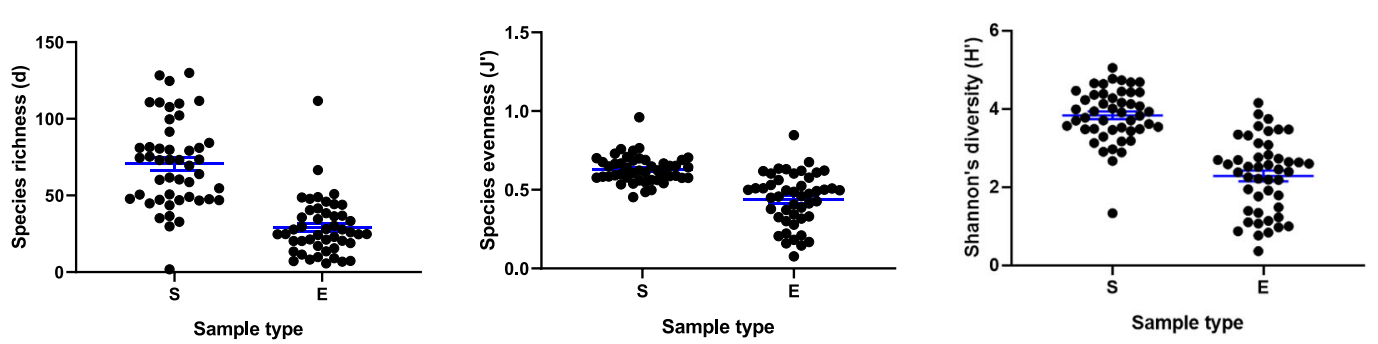

B

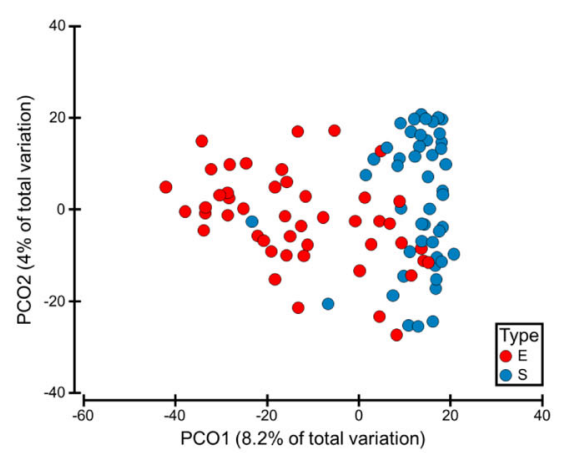

D

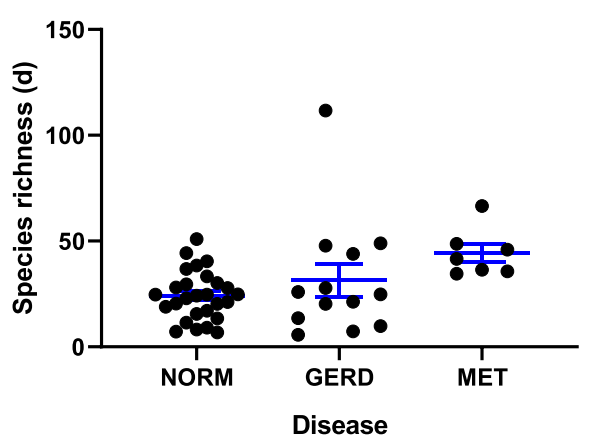

$\mathbf{F}$

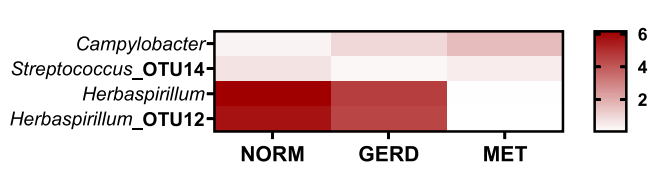

G

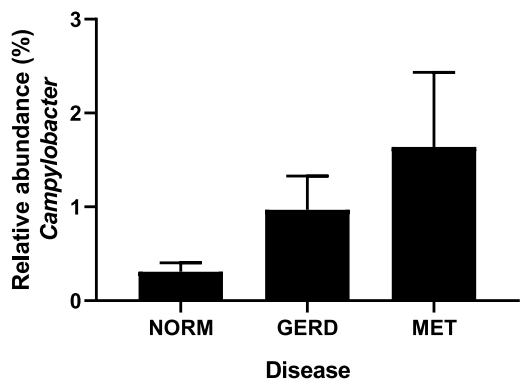

C

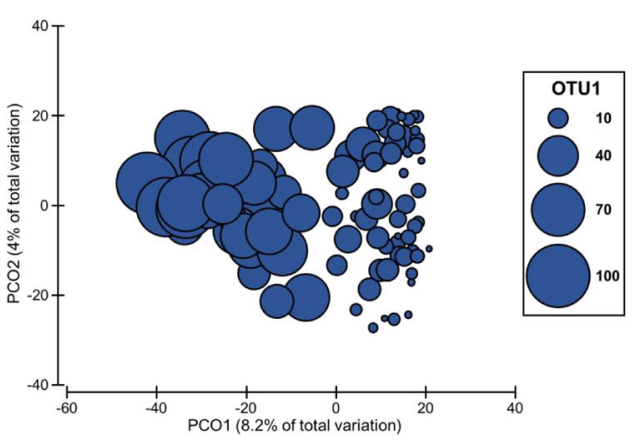

E

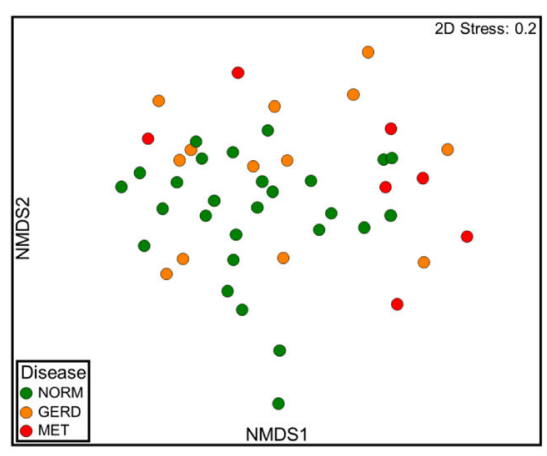

H

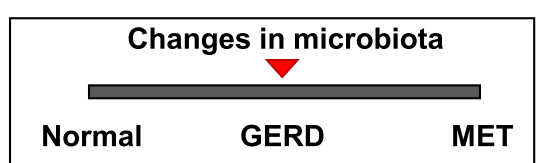

I

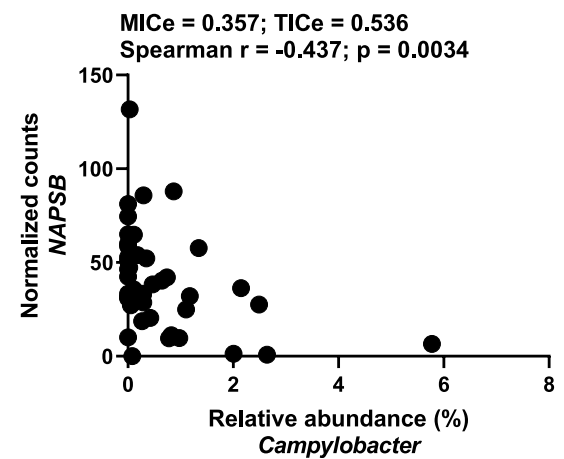

Fig. 2 (See legend on next page.) 


\section{(See figure on previous page.)}

Fig. 2 The saliva and esophageal microbiota in subjects with GERD and metaplasia. Full-length 16S rRNA amplicon sequencing was performed on a PacBio platform. A Comparison of alpha diversity measures between saliva (S) and esophageal (E) samples. Significant differences between sample types (Richness: Pseudo-F: 69.7, $P=0.001$, df = 92; Evenness: Pseudo-F: 48.7, $P=0.001$, df = 92; Shannon's diversity: Pseudo-F: 83.2, $P=$ $0.001, \mathrm{df}=92$ ) were observed across all measures using linear models that corrected for age, sex, PPI, BMI, reflux symptoms, and disease. $\mathbf{B}$ Principal coordinate analysis of Bray-Curtis resemblance matrix generated from square-root-transformed pOTU relative abundances. Distancebased linear models corrected for all variables identified significant differences in composition between sample types (Pseudo-F: 5.1, $P=0.001, \mathrm{df}$ = 92). $\mathbf{C}$ The same principal coordinate analysis as above incorporating the relative abundance of Streptococcus pOTU1. D Esophageal species richness stratified according to disease. E Non-metric multidimensional scaling plot of Bray-Curtis resemblance matrix generated from squareroot-transformed OTU relative abundances. Only esophageal samples were plotted. F Heatmap of genera and OTUs identified by LEfSe to be significantly different (LDA > 3) between normal and GERD (Additional file 1: Table S9) or normal and MET (Additional file 1: Table S10). G Mean relative abundance of Campylobacter stratified according to disease. Errors are SEM. $\mathbf{H}$ Significant changes in the esophageal microbiota occur by the time of diagnosis of GERD. I A significant co-exclusion relationship between the relative abundance of Campylobacter and NAPSB was present. Non-parametric correlations were identified through MINe and confirmed with Spearman's correlation

$\mathrm{m}_{12}=0.206, P=0.001$ ) were observed (Additional file 2: Figure S6A). The matrices were also found to be significantly correlated (Spearman Rho: 0.309, $P=0.004$ ). A similar significant concordance was seen between the transcriptome and the microbiome (Illumina) data sets (PCoA: $\mathrm{m}_{12}=0.264, P=0.001 ; \mathrm{dbRDA}: \mathrm{m}_{12}=0.175, P$ $=0.001$ ) (Additional file 2: Figure S6B) but the correlation did not reach significance (Spearman Rho: 0.083, $P$ $=0.19)$. To confirm the validity of the relationships, $a$ random matrix was generated, and no significant correlation was observed against the transcriptome data set (Spearman Rho: $-0.041, P=0.727$ ).

Next, the associations between the strongest microbial signature in our data (relative abundance of Campylobacter) and the host transcriptome were assessed. A strong correlation between the relative abundance of Campylobacter (PacBio) and the abundance of active MAST cells was observed (Pearson $\mathrm{R}=0.703$, 95\% CI $0.510-0.828, P=0.0001$, FDR $=0.002)$. Non-parametric correlations between transcript counts and the relative abundances of Campylobacter (PacBio, Additional file 1: Table S12) and Campylobacter iOTU36 (Additional file 2: Figure S7A; Additional file 1: Table S13) were also calculated, and a range of exponential relationships with molecules were identified (Additional file 2: Figure S7B). Notably, a significant co-exclusion relationship between the relative abundances of Campylobacter taxa and NAPSB were present (both of our data sets, Fig. 2I; Additional file 2: Figure S7C), this pseudogene being found to be consistently significantly downregulated in GERD and MET when compared to NORM samples (Fig. 1E).

\section{Targeted culture identifies Campylobacter isolates with increased capacity to survive in primary macrophages} In parallel to the sequencing strategies, several culture strategies for the isolation of microaerobic and anaerobic bacteria were implemented on the same saliva and esophageal mucosal samples, including a filter strategy [34] for the isolation of Campylobacter species. Eleven
Campylobacter isolates were grown from saliva samples of normal $(n=3)$, GERD $(n=4)$, and $\operatorname{MET}(n=4), 10$ of which were putatively identified as Campylobacter concisus and 1 as Campylobacter rectus on 16S rRNA amplicon sequencing with Sanger sequencing.

Despite the fact that the salivary and esophageal microbiotas were found to be distinct in profile in this cohort, our source tracking analysis revealed that the majority of the detected esophageal bacteria did originate from the oral cavity. This would indicate that strains isolated from the patient's saliva were good representatives of those found in their esophagus. Thus, the proinflammatory potential of these isolates was assessed through co-culture with primary human macrophages and measurement of the levels of 34 cytokines and chemokines at two time points ( 4 and $18 \mathrm{~h}$ ). The capacity of these isolates to survive within primary macrophages was also determined at $4 \mathrm{~h}$, given that a co-exclusion relationship between Campylobacter and lysosomal transcripts was identified.

While significant differences in cytokine and chemokine production was observed between non-infected and infected cells ( $4 \mathrm{~h}: t=4.36, P=0.016, \mathrm{df}=11 ; 18 \mathrm{~h}: t=$ $8.01, P=0.013$, df $=11)$, and between 4 and $18 \mathrm{~h}$ infection time points $(t=8.56, P=0.001, \mathrm{df}=20)$, no differences were found across disease groups $(p>0.67$ for all) (Fig. 3A). In contrast, substantial differences in the capacity of the isolates to survive within primary macrophages were identified (Fig. 3B). Two isolates (C. concisus ESOS44-1 and C. rectus ESOS44-4) from a patient with MET and three isolates from patients with GERD (C. concisus ESOS14-1, ESOS15-1, and ESOS33-1) all had intracellular levels greater than $1 \%$. No isolates from NORM showed high intracellular levels $(0 \%, 0 \%$, and $0.082 \%)$. Notably, a strain previously isolated from a patient with Crohn's disease (UNSWCD), which had a strong capacity to invade intestinal epithelial cells [46], showed a weaker capacity $(0.27 \%)$ to survive in primary macrophages. 
A

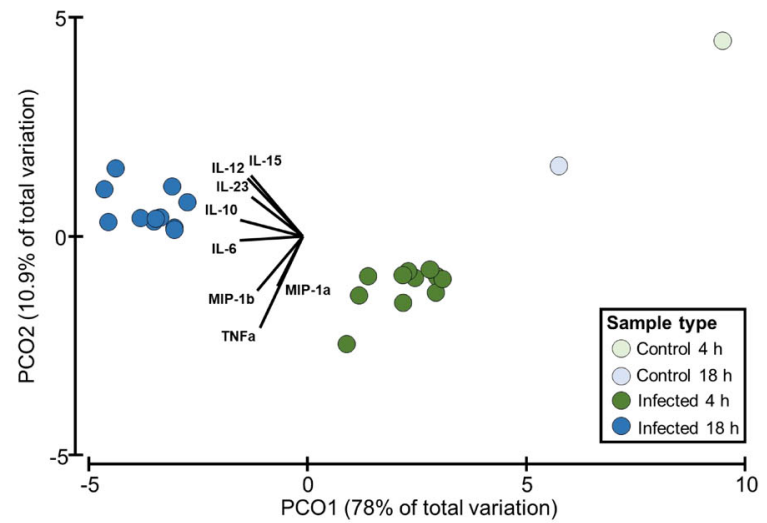

C

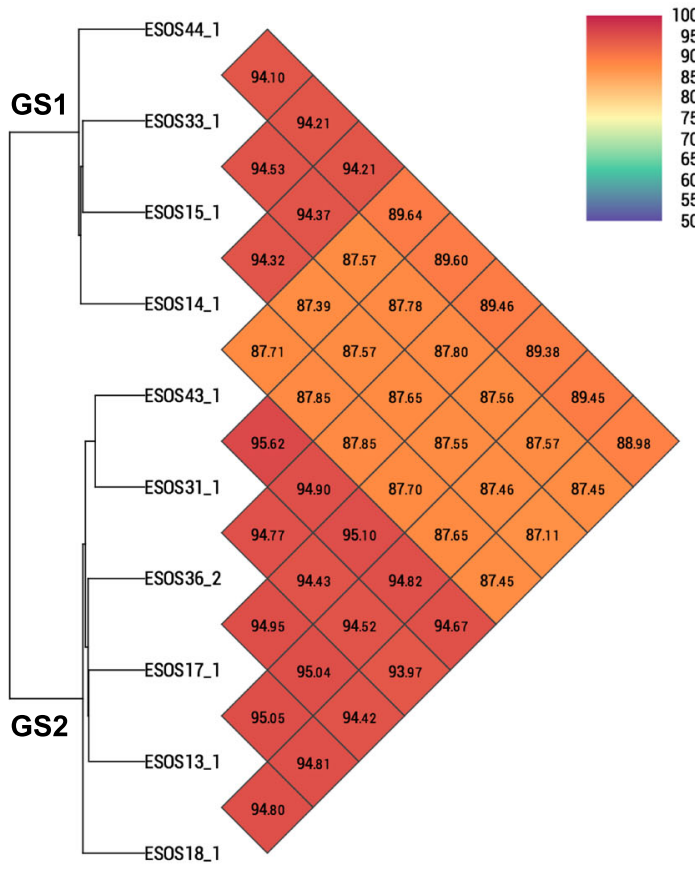

$\mathbf{F}$

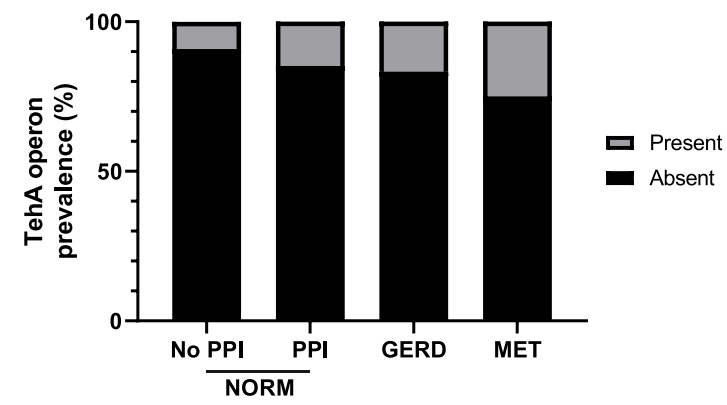

B

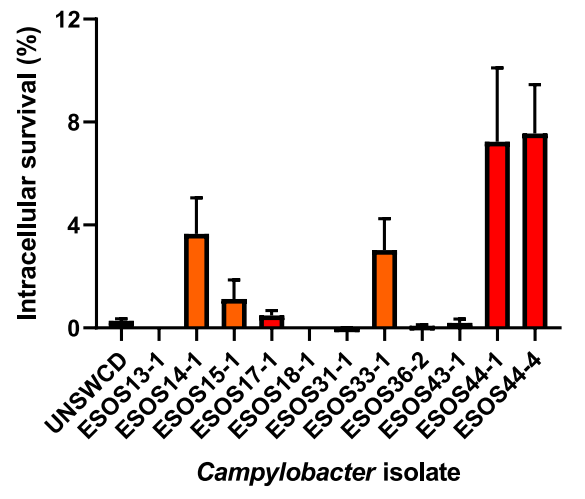

D

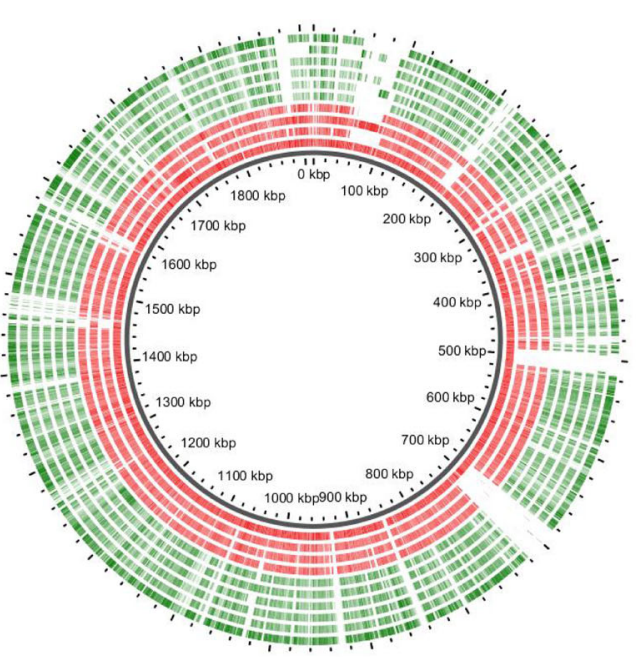

E $\begin{array}{ccc}\text { TehA } & \text { Crp/Fnr } & \text { Oxidoreductases Hypothetical } \\ 00813 & 00814 & 0081500816 \quad 0081700818\end{array}$

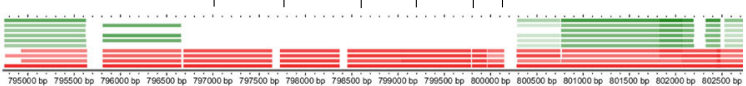

G

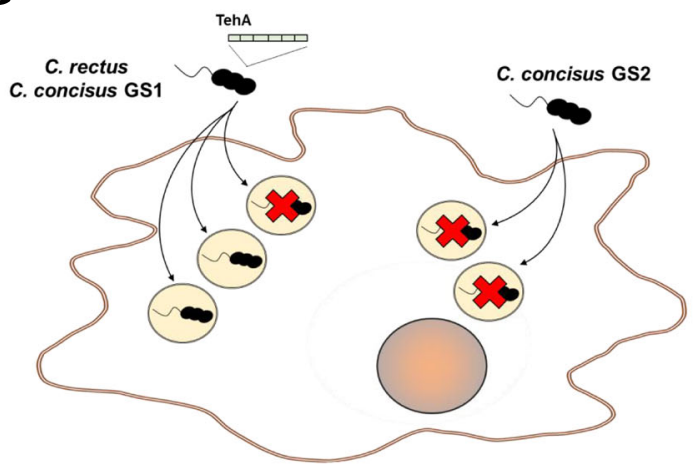

Fig. 3 (See legend on next page.) 
(See figure on previous page.)

Fig. 3 Virulence and genomic differences across Campylobacter isolates from patients with GERD and metaplasia. A Principal coordinate analysis of the levels of 34 cytokines and chemokines produced by primary macrophages upon infection with Campylobacter isolates. Macrophages were infected with 10 different $C$. concisus and 1 C. rectus isolate from this patient cohort. Data was $\log (x+1)$ transformed and a Euclidean distance matrix generated. Vectors were generated using a correlation value of 0.3 for both Pearson and Spearman correlations. B Intracellular levels of Campylobacter isolates within primary human macrophages. Levels were calculated using a gentamicin protection assay at MOI 100. ESOS44-1 and ESOS44-4 were significantly different to all other isolates except for ESOS14-1, ESOS33-1, and each other (ANOVA, Tukey's). No other comparisons were significant. Red: isolates from MET, orange: from GERD, and green: from NORM. C Average nucleotide identity across the 10 C. concisus isolates' genomes. A separate analysis including C. rectus showed an ANI of 71.02-71.39\%. D Circular representation of the 10 C. concisus genomes using GView. GS1 strains, red; GS2 strains, green. E Syntenic proteins unique to GS1 strains as compared to GS2 strains, including the tellurite resistance protein TehA. $\mathbf{F}$ Prevalence of the TehA operon in shotgun metagenomics data of esophageal mucosal brushings from patients in the early stages of the EAC cascade. Reads aligning to the TehA operon within the data were identified using BWA-MEM. G C. concisus GS1 isolates and C. rectus have enhanced intracellular survival in primary macrophages when compared to C. concisus GS2 isolates, and the former can be delineated by the presence of six syntenic proteins including TehA

\section{C. concisus genomospecies 1 has increased intracellular survival in primary macrophages and shares a set of six syntenic proteins with $C$. rectus}

To identify the possible genetic basis for increased intracellular survival in macrophages by certain Campylobacter isolates, the genomes of all 11 strains were sequenced using both PacBio and Illumina chemistry. Unsupervised comparative genomics through average nucleotide identity (ANI) was applied on the $10 \mathrm{C}$. concisus genomes, and two unique clusters were identified, with isolates found to have increased survival $(>1 \%)$ clustering together (Fig. 3C). These clusters corresponded to the two C. concisus genomospecies (GS) previously identified [47], with GS1 being that which survives within primary macrophages.

Supervised comparative analyses identified 25 proteins unique to our four GS1 isolates when compared to the other six GS2 isolates, a substantial portion of which were involved in redox reactions, functions highly relevant in macrophage pathogen elimination machinery (Fig. 3D; Additional file 1: Table S14). Intriguingly, within these 25 proteins was a group of six proteins found conserved in synteny within the genome of the four GS1 isolates (Fig. 3E), including tellurite resistance protein TehA, a Crp/Fnr family transcriptional regulator, two oxidoreductases, and two DUF2892 domaincontaining proteins. The whole syntenic group was also found to be highly conserved in C. rectus ESOS44-4 (average similarity 69-82\%). Further, analyses of the presence of the 25 unique proteins across the 224 available C. concisus genomes showed that 88 strains had good conservation of these proteins, with the operon of interest containing TehA delineating this separation effectively (tblastn, Additional file 1: Table S14). This clustering was also concordant with clustering according to GS.

To test the prevalence of the TehA operon and TehA only within the esophageal microenvironment across the disease cascade, shotgun metagenomics data of esophageal brushings [10] were searched for reads matching these regions using BWA-MEM. A stepwise increase in the prevalence of reads mapping to these two regions was observed across the disease cascade (Fig. 3F; Additional file 2: Figure S8A); however, the increasing trend did not reach statistical significance (TehA operon: $\chi^{2}=1.44, P=0.23$; TehA: $\chi^{2}=1.53, P=0.22$; chi-square test for trend).

In summary, C. concisus GS1 isolates and C. rectus have enhanced intracellular survival in primary macrophages (Fig. 3G) and can be delineated by the presence of six syntenic proteins including TehA.

\section{Campylobacter isolates from a patient with metaplasia possess additional genetic elements that improve fitness} In addition to the increased intracellular survival of GS1 isolates, an even higher capacity to survive was noted for one isolate from a patient with BAR (ESOS44-1), and this increased capacity was shared with $C$. rectus ESOS44-4, isolated from the same patient (Fig. 3B). To identify genes unique to ESOS44-1 and shared by ESOS44-4, first, comparative genomic analyses were performed against the other nine $C$. concisus isolates, with a total of 60 proteins found to be unique to ESOS44-1 (Fig. 4A; Additional file 1: Table S15). A substantial number of these unique proteins were found in syntenic groups, with two large regions corresponding to Prophage CP4-57 (region I) and proteins involved in lipid A biosynthesis (region II) (Fig. 4A; Additional file 1: Table S15). Lipid A modifications are important in modulating outer-membrane permeability, resistance to antimicrobial peptides, and host recognition [48], whereas prophage CP4-57 has been implicated in acid resistance [49], biofilm formation, and lactate utilization [50], suggesting that these elements may contribute to enhanced fitness of this isolate. An additional group of 5 syntenic proteins (01601-01605; Fig. 4B; Additional file 1: Table S15) was also unique to ESOS44-1, with domain analysis showing 01601 to contain the $N$-terminal part of LprI, a protein within Mycobacterium tuberculosis that detoxifies lysozyme [51]. 


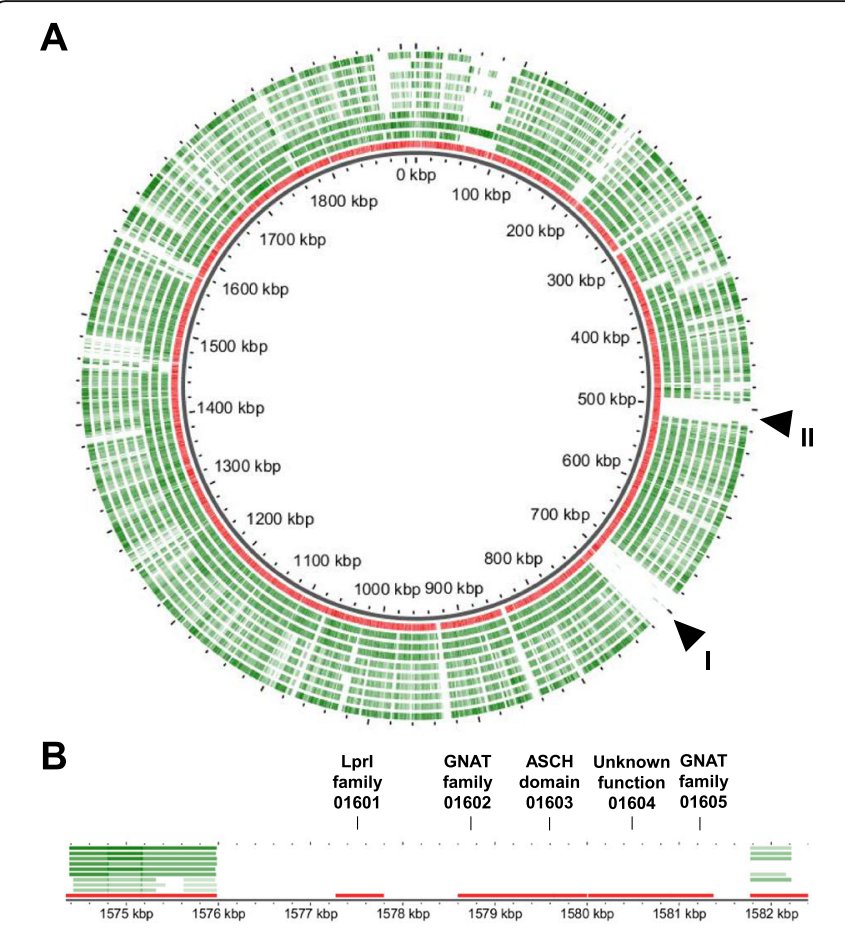

C

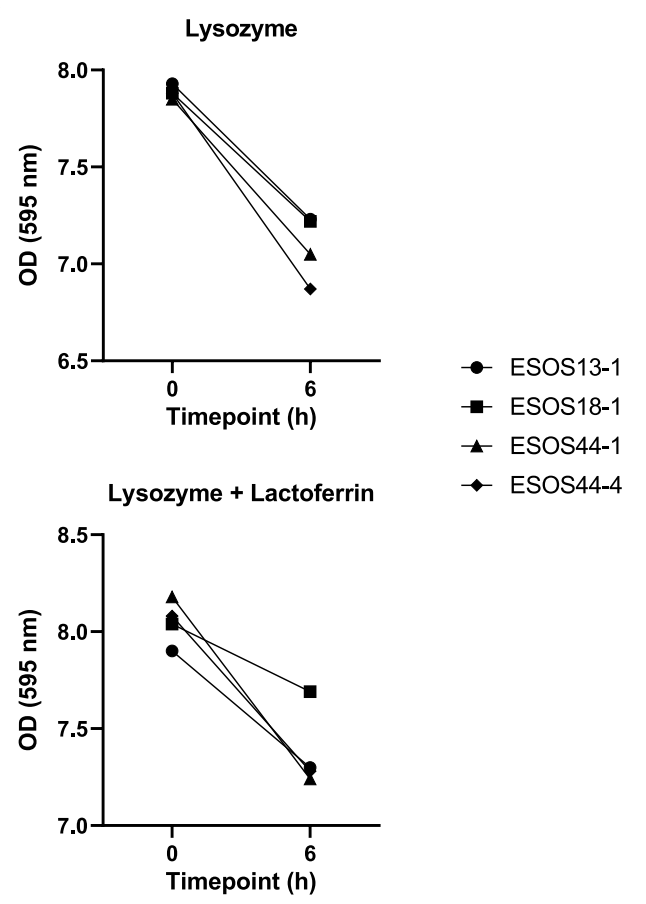

D

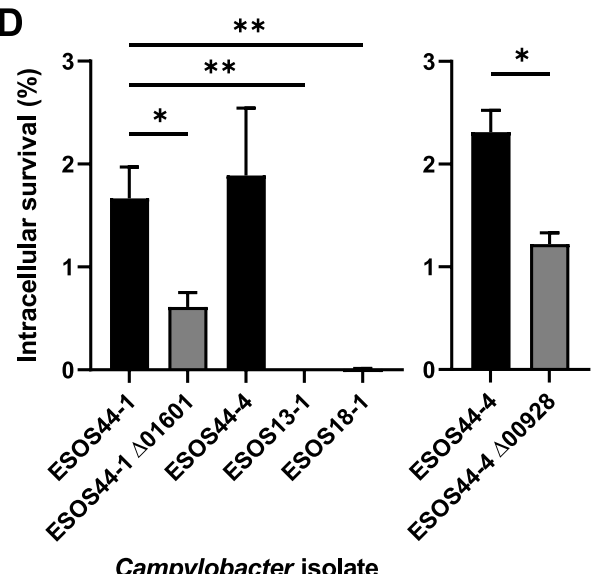

G

\begin{tabular}{|c|l|c|}
\hline Protein & Protein name & Score \\
\hline CNN2 & Calponin 2 & \\
\hline GLIPR2 & GLL Pathogenesis Related2 & \\
\hline PLD3 & Phospholipase D3 & \\
\hline CTSS & Cathepsin S & \\
\hline LGALS9 & Galectin 9 & \\
\hline TAF15 & TATA-Box Binding Protein Associated Factor15 & \\
\hline UQCRH & Ubiquinol-CytochromeC Reductase Hinge Protein & \\
\hline PTBP1 & Polypyrimidine Tract Binding Protein 1 & \\
\hline ANXA2 & Annexin A2 & \\
\hline LGMN & Legumain & \\
\hline
\end{tabular}

E

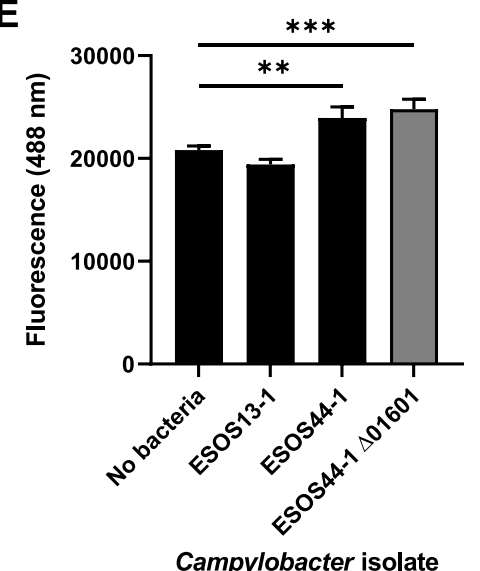

H

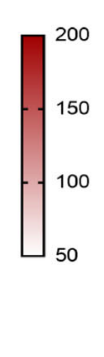

F
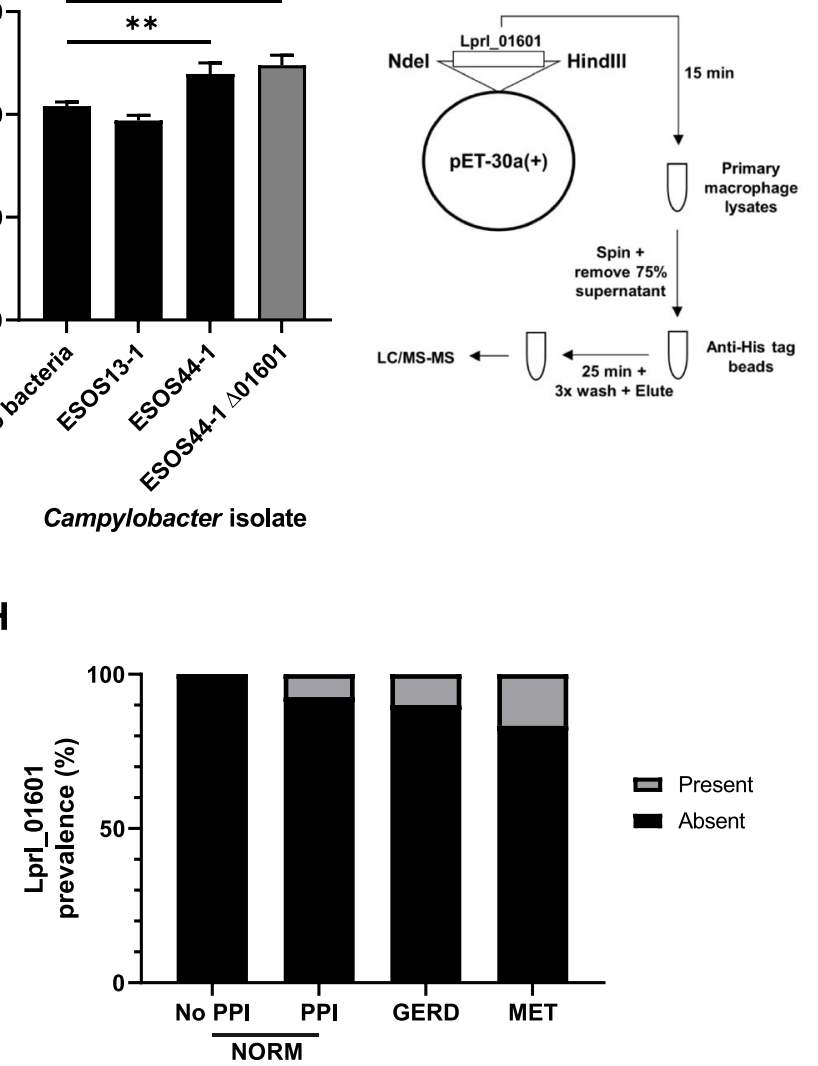

Fig. 4 (See legend on next page.) 


\section{(See figure on previous page.)}

Fig. 4 Characteristics of C. concisus ESOS44-1 and ESOS44-4 compared to other C. concisus isolates from this cohort. A Circular representation of the 10 C. concisus genomes using GView. ESOS44-1, red; other isolates, green. Region I: Prophage CP4-57; Region II: lipid A biosynthesis. B Syntenic proteins unique to ESOS44-1 as compared to other isolates, including Lprl_01601. C Susceptibility of representative Campylobacter isolates to lysozyme as well as lysozyme and lactoferrin. Experiments were repeated in triplicate and representative results were presented. D Intracellular levels of representative wild-type Campylobacter isolates and the $\Delta$ Lprl_01601 mutant within primary human macrophages. Levels were calculated using a gentamicin protection assay at MOI 100. ESOS44-1 was significantly different to all other strains except for ESOS44-4 (ANOVA, Dunnett's; ${ }^{*} P<0.05,{ }^{* *} P<0.01$ ). Decreased intracellular levels of ESOS44-4 $\Delta$ Lprl_00928 as compared to the wild-type isolate were validated in primary macrophages from another donor (Welch's $t$-test; ${ }^{*} P<0.05$ ). E Acidification of lysosomes following infection with wild-type C. concisus isolates and the mutant strain. Fluorescence was measured following the addition of LysoTracker Green. ESOS44-1 and the $\Delta$ Lprl_01601 mutant were both significantly different to the negative control (ANOVA, Dunnett's; ${ }^{* *} P<0.01$, *** $P<0.001$ ). $\mathbf{F}$ Experimental procedure for Lprl_01601 recombinant expression and immunoprecipitation following incubation with primary macrophage lysates. G Human proteins that co-immunoprecipitated with Lprl_01601. Proteins are arranged according to combined mascot score from two IP and LC/MS-MS experiments. H Prevalence of Lprl_01601 in shotgun metagenomics data of esophageal mucosal brushings from patients in the early stages of the EAC cascade. Reads aligning to Lprl_01601 within the data were identified using BWA-MEM

Next, the presence of these 60 proteins unique to ESOS44-1 against the other $C$. concisus isolates was investigated in $C$. rectus ESOS44-4. A flavin reductase (00170), three proteins within region I, and notably, the putative lysozyme-detoxifying LprI homolog 01601 (00928 in C. rectus), were conserved in the proteome of ESOS44-4. Thus, the susceptibility of a subset of the isolates to lysozyme was then tested, and no differences were found between strains possessing LprI_01601 and those that do not (Fig. 4C). A modified lysozyme susceptibility protocol for Gram-negative bacteria including lactoferrin [52] was also applied, and again, no differences were observed (Fig. 4C), suggesting this protein may have other activities beyond lysozyme detoxification. Next, a mutant lacking LprI_01601 was generated using allelic exchange through natural transformation, and the ability of the mutant to survive intracellularly in primary macrophages was compared to the wild-type strain as well as representative GS2 strains. A significant decrease ( 2.7-fold, $p=0.020$; ANOVA-Dunnett's test) in intracellular levels of the LprI_01601 mutant was found when compared to the wild-type ESOS44-1 (Fig. 4D). This decrease in intracellular levels was validated in the ESOS44-4 LprI_00928 mutant (Fig. 4D), showing a 1.9-fold decrease in levels. The addition of latrunculin A, an inhibitor of phagocytosis, abolished intracellular bacteria in both wild-type and mutant, indicating that internalization was completely reliant on phagocytosis and not transcellular invasion. Given this, lysosome acidification was tracked upon infection using LysoTracker. Acidification was more evident at $4 \mathrm{~h}$ in primary macrophages infected with ESOS44-1 wild-type and mutant strains, and this was not observed in the representative GS2 strain ESOS13-1 (Fig. 4E). This suggested that not only improved intracellular fitness but also increased phagocytosis contribute to the ESOS44-1 phenotype.

To provide insights into possible interacting partners of LprI_01601 within the host, recombinant LprI_01601 was used as a bait to pull down binding partners from primary human macrophage cell lysates (Fig. 4F). Ten human proteins that passed a strict filtration cutoff were identified, including the three lysosomal proteins cathepsin S, legumain, and galectin 9 (Fig. 4G), supporting a potential role for this bacterial protein within the host lysosome. Of note, the genes encoding cathepsin $\mathrm{S}$ and galectin 9, but not legumain were significantly upregulated in the transcriptome of patients with MET in our cohort (Additional file 1: Table S2).

The prevalence of LprI_01601 was then assessed in the shotgun metagenomics data set [10], similar to what was performed for TehA. A stepwise increase in prevalence was found (Fig. $4 \mathrm{H}$ ), and the relationship with the disease cascade showed a borderline non-significant trend for this protein $\left(\chi^{2}=3.27, P=0.071\right.$; chi-square test for trend). Next, to conclusively detect the presence of Campylobacter with increased intracellular fitness, the prevalence of either "LprI_01601 or TehA" as well as either "LprI_01601 or the TehA operon" was also assessed in this data. A significant trend was identified for LprI 01601/TehA $\left(\chi^{2}=4.52, P=0.033\right.$; Additional file 2 : Figure S8B) and a borderline non-significant trend identified for $01601 /$ TehA operon $\left(\chi^{2}=2.69, P=0.10\right.$; Additional file 2: Figure S8C).

These findings show that certain C. concisus strains have increased intracellular fitness arising from specific genomic features, some of which are shared by $C$. rectus. These features increased in prevalence in shotgun sequencing data from diseased samples from an independent cohort.

\section{Campylobacter isolates with increased intracellular survival have differential effects on primary esophageal epithelial cells}

To investigate the effects of these different Campylobacter isolates on epithelial cells, primary esophageal epithelial cells were cultured and infected with representative isolates GS2 (ESOS13-1, ESOS18-1), GS1 (ESOS14-1, ESOS33-1, ESOS44-1), and C. rectus ESOS44-4. Bulk 
shotgun sequencing of RNA from the infected cells was performed and compared to that from non-infected cells. C. concisus isolates had a modest effect $(n=21$ to 83 genes) when compared to $C$. rectus ( $n=1028$ genes)

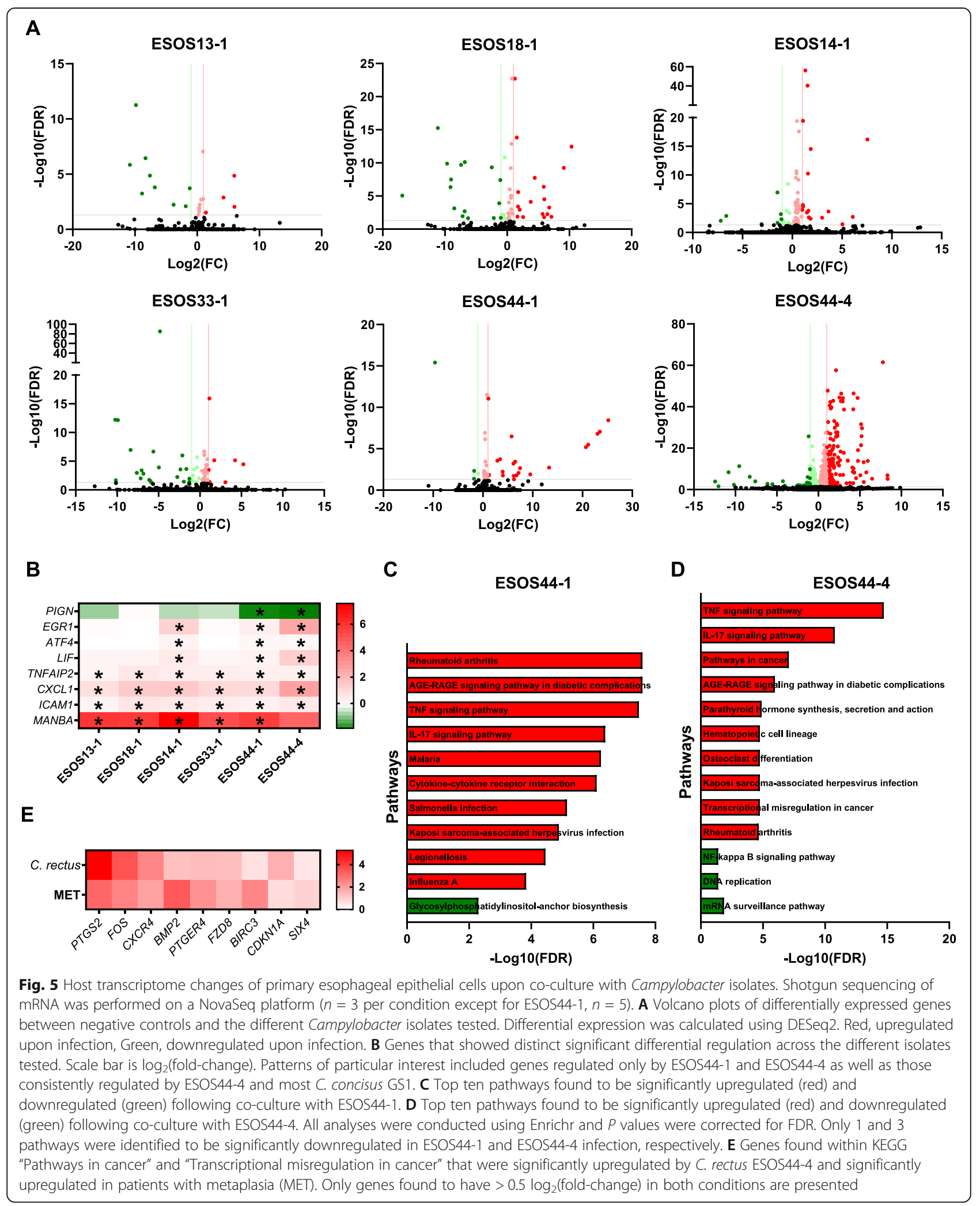


(Fig. 5A; Additional file 1: Tables S16-21). Several genes were found to be significantly regulated across all isolates tested including TNFAIP2, CXCL1, and ICAM1, while $M A N B A$ (Beta-mannosidase) was found to be significantly upregulated in all C. concisus isolates (Fig. 5B). The genes EGR1, ATF4, and LIF were upregulated by most GS1 isolates and C. rectus whereas PIGN was downregulated only in the isolates with especially high intracellular survival (ESOS44-1 and ESOS44-4) (Fig. $5 \mathrm{~B})$. Notably, EGR1 was one of the 4 genes to be consistently, significantly upregulated in patients with GERD and MET (Fig. 1E).

Pathway analysis identified a substantial number of pathways commonly regulated by the different C. concisus isolates including several inflammatory pathways such as TNF and IL-17 signaling; however, "Glycosylphosphatidylinositol (GPI)-anchor biosynthesis" was uniquely downregulated by ESOS44-1 (Fig. 5C). Notably, while $C$. rectus ESOS44-4 also upregulated TNF and IL17 signaling, it significantly upregulated "Pathways in cancer" as well as "Transcriptional misregulation in cancer" (Fig. 5D). Genes within these two pathways that were upregulated by $C$. rectus were then assessed against genes upregulated in patients with MET (Fig. 1D), and 9 genes were found to have a significant $\log _{2}$ (fold-change) $>0.5$ in both conditions when compared to their relevant controls (Fig. 5E).

These results demonstrated that Campylobacter isolates with high intracellular levels could regulate a range of inflammatory pathways in primary esophageal epithelial cells, as well as modulate genes identified to be differentially expressed in patients with GERD and MET. Further, C. rectus significantly upregulated pathways of high relevance to cancer development.

\section{Discussion}

Little is known about the esophageal microenvironment when compared to the oral and lower gut. Here, we first assessed the host transcriptome and found that while some changes occur in GERD, most of the identified changes in expression, splicing, circular RNAs, and fusion events occur either in the transition from GERD to MET, or in MET. We then compared matched oral and esophageal microbiomes in the same cohort and confirmed that the esophageal microbiota is distinct, but most esophageal species can be source traced to the oral cavity. We identify changes in the esophageal microbiota with GERD and MET, with enrichment of Campylobacter found to be a consistent signature that starts in GERD. The microbiome and transcriptome profiles within the same samples were correlated, and the relative abundance of Campylobacter was associated with molecules associated with recognition of bacteria and the lysosome. In parallel, we isolated Campylobacter from the same patients and showed that isolates from patients with disease were more likely to have genomic features associated with intracellular fitness within primary immune cells. These genomic features were found to progressively increase in prevalence with disease progression in shotgun metagenomics data of esophageal brushings from an independent cohort. Co-culture of isolates with primary esophageal epithelial cells showed that most isolates that can survive intracellularly upregulated $E G R 1$, which was found to be consistently upregulated in GERD and MET. C. rectus ESOS44-4 was also shown to upregulate pathways of high relevance to cancer development.

Bulk transcriptome profiling was suggestive of patients with metaplasia having higher levels of active mast cells, in contrast to higher levels of resting mast cells in patients with normal esophagi and GERD. This is concordant with immunohistochemical analysis of esophageal biopsies from patients with BAR which showed higher numbers of mast cells when compared to patients with GERD [53]. Interleukin-13, a cytokine commonly produced by mast cells, has been robustly linked to hyperplasia and mucus hypersecretion in airway epithelial cells $[54,55]$. The transcriptome of patients with metaplasia was also found to resemble that of colonic cells, in line with the pathological transformation from a stratified epithelium to intestinal-like columnar cells. Robust markers of metaplasia in our study included cathepsin E (CTSE), which has been associated with gastric and colon cancers and previously shown to be upregulated in patients with BAR and EAC [56], as well as the anion/bicarbonate channel CFTR and PDZ-domain-containing mucins MUC17, MUC3A, and MUC12. The link between CFTR and MUC3 has been suggested to be involved in the mucus phenotype of certain diseases [57].

We also identified several markers that were consistent across patients with GERD and MET when compared to patients with normal biopsies. FREM2, a protein associated with alteration of the extracellular matrix to enable cell migration and rearrangements [58], showed a progressive increase from NORM to GERD to MET. This protein has also been suggested to facilitate IL- $1 \beta$ signal transduction through stabilizing IL-1R1 [59], an inflammatory pathway strongly linked to the EAC cascade [9]. Another marker of interest was the circular RNA transcript of CUL6, a component of the SCF ubiquitin ligase, which significantly decreased in prevalence in both GERD and MET and has been shown to promote defense against microbial species [60]. Of most relevance, however, was the progressive decrease in expression of NAPSB, a lysosomal aspartic protease related to napsin A (NAPSA) which has pseudogenized in most human populations $(0-4.5 \%$ retain the active allele) [61]. 
We observed an increase in relative abundance of Campylobacter taxa at the stage of GERD that continued in metaplasia, regardless of the cohort studied or chemistry used. We also observed a significant increase in prevalence of a Campylobacter taxon of interest in disease. Several Campylobacter species such as $C$. rectus and $C$. concisus are members of the human oral microbiota but have been associated with various diseases. These bacteria are normally described as mucosa associated as they can traverse the mucus layer of the gastrointestinal tract efficiently [62]. They are also phylogenetically related to Helicobacter pylori, a causative agent of gastric cancer [63]. Their enrichment appeared to be at the expense of Streptococcus and Herbaspirillium. While the latter is considered a common extraction kit contaminant, viable Herbaspirillium have been previously isolated from the respiratory tract of humans [64]. Intriguingly, the relative abundance of Campylobacter was found to be in a co-exclusion relationship with $N A P S B$, one of the five genes consistently regulated in GERD and MET, which suggests Campylobacter may have some intracellular component to its lifestyle in vivo.

Isolation of Campylobacter species from this cohort confirmed intracellular survival within primary macrophages was a highly relevant characteristic across strains, with a genome-wide analysis identifying a genomic basis to this feature. Specifically, C. concisus isolates classified as GS1 had higher survival, and proteins unique to GS1 were enriched for oxidoreductases. A unique group of six syntenic proteins including tellurite resistance protein TehA was a key differentiating feature across these groups and was also found in C. rectus ESOS44-4. TehA has been found to contribute to the intracellular survival of Corynebacterium diphtheriae in epithelial cells [65] and resistance to antiseptics and disinfectants in Escherichia coli [66]. Of note, bacteria in the tumor microenvironment have been recently reported to consist mostly of species that have an intracellular niche in different tumor cell types including immune cells [67], with Campylobacter species being members of a polymicrobial signature associated with colorectal cancer [68].

A deeper analysis of one GS1 C. concisus isolate from a patient with MET that had higher relative survival than other GS1 isolates found one large unique genomic region linked to lipid A modifications as well as a prophage region previously linked to acid resistance [49]. A higher resistance to acid could contribute to this isolate having higher survival despite leading to higher lysosomal acidification upon infection. Further comparative analysis taking into consideration $C$. rectus isolated from the same patient pointed towards another group of syntenic proteins that included a protein containing a domain from LprI, a Mycobacterium protein that detoxifies lysozyme [51]. Knock-out of LprI_01601 did lead to decreased intracellular survival, and this finding was validated with the C. rectus LprI_00928 mutant, but the mechanism appeared to be independent of lysozyme. Indeed, on preliminary screen, recombinant LprI_01601 did not appear to interact with human lysozyme but was pulled down along with the lysosomal cysteine protease cathepsin S, galectin 9 [69], and legumain, of which the former is a substrate [70]. Notably, cathepsin $S$ and galectin 9 were upregulated in patients with MET in our cohort. Lower cathepsin $\mathrm{S}$ has been previously linked to higher intracellular survival of Mycobacterium tuberculosis in macrophages [71], and this autophagy-associated protein has been linked to inflammatory and infectious periodontal disease [72]. However, the influence of knock-out of LprI_01601 on the remainder of the syntenic proteins cannot be discounted. Two of these proteins are acetyltransferases, a function that influences bacterial virulence [73, 74], most notably, the capacity of C. jejuni for lysozyme resistance and intracellular survival in macrophages through acetylation of its peptidoglycan [57].

Of significance, the identified signatures delineating Campylobacter with increased intracellular fitness (TehA operon and LprI_01601) increased in prevalence with disease progression (normal on PPIs, GERD, then MET) in shotgun metagenomics data from esophageal brushings of patients with GERD and MET, supporting a possible role for Campylobacter isolates possessing these proteins in esophageal disease. These results are of biological relevance given the unsupervised and unbiased nature of this analysis.

Co-culture of representative GS2 and GS1 isolates as well as $C$. rectus ESOS44-4 with primary esophageal epithelial cells showed that all isolates could induce TNF and IL-17 signaling but only the GS1 isolates (ESOS14-1 and ESOS44-1) and C. rectus led to an upregulation of $E G R 1$, a gene that was also upregulated in patients with GERD and MET. This gene has been reported to be potential biomarker of BAR with low-grade dysplasia and EAC [75], and to have high expression in precancerous lesions of the stomach and esophagus [76, 77]. These isolates also upregulated LIF, an IL-6 family cytokine associated with the lack of efficacy of neoadjuvant therapy in EAC [78]. Another gene of interest is PIGN, found to be downregulated by the two isolates ESOS44-1 and ESOS44-4 with increased intracellular levels. PIGN has been reported to be a chromosomal instability suppressor in cancer whose silencing can lead to DNA replication stress and associated damage [79]. Importantly, C. rectus infection significantly upregulated $>50$ genes associated with cancer development, and a range of these were commonly upregulated in patients with MET as well. Given that $C$. rectus is substantially lower in prevalence in the human oral cavity than the commonly detected $C$. 
concisus, the specific consequences of its presence within the microbiota on the host need to be investigated further.

Our study had some limitations. This includes the size of the prospective cohort which we attempted to overcome by screening for signatures in a previously published cohort. Future studies should validate the presence of these signatures in larger cohorts and take into account additional variables such as smoking and diet. Further, we did not include patients with EAC as our design focused on etiological factors of metaplasia. Additional studies should investigate if these signatures are present in EAC, or if similar to gastric cancer, microbial etiological factors disappear.

\section{Conclusions}

Taken together, our work utilizes multi-omics strategies to identify strain-level signatures of relevance to esophageal disease, pointing towards a role for C. rectus and some C. concisus GS1 with increased intracellular fitness in primary macrophages. Our work highlights the utility of an unbiased systems approach in transitioning complex microbiome signatures from correlation to causation in situations where animal models are not readily available.

\section{Abbreviations}

ESCC: Esophageal squamous cell carcinoma; EAC: Esophageal adenocarcinoma; GERD: Gastro-esophageal reflux disease; BAR: Barrett's esophagus; MET: Metaplasia; NORM: Normal; BMI: Body mass index; OTU: Operational taxonomic unit; ANI: Average nucleotide identity

\section{Supplementary Information}

The online version contains supplementary material available at https://doi. org/10.1186/s13073-021-00951-6.

Additional file 1: Table S1. Transcripts differentially expressed between normal and GERD samples. Table S2. Transcripts differentially expressed between normal and MET samples. Table S3. Pathway analysis of differentially expressed transcripts. Table S4. Genes identified with possible differences in splicing events. Table S5. Intron retention and exon skipping events found between disease groups. Table S6. Circular RNAs tested for differential prevalence within disease groups. Table S7. Gene fusion events identified within the esophageal samples. Table S8. Per taxa differences between saliva and esophageal samples. Table $\mathbf{S 9 .}$ Per taxa differences between normal and GERD esophageal samples. Table S10. Per taxa differences between normal and MET esophageal samples. Table S11. Per taxa differences between normal and disease esophageal samples. Table S12. MINE analysis between Campylobacter and transcripts. Table S13. MINE analysis between Campylobacter iOTU36 and transcripts. Table S14. Conservation of proteins unique to GS1 C. concisus isolates. Table S15. Proteins unique to C. concisus ESOS44-1. Table S16. Differential expression between control and Campylobacter ESOS13-1. Table S17. Differential expression between control and Campylobacter ESOS14-1. Table S18. Differential expression between control and Campylobacter ESOS18-1. Table S19. Differential expression between control and Campylobacter ESOS33-1. Table S20. Differential expression between control and Campylobacter ESOS44-1. Table S21. Differential expression between control and Campylobacter ESOS44-4.

Additional file 2: Figure S1. Splicing events within the esophageal transcriptome. Figure S2. Exon skipping events within the esophageal transcriptome. Figure S3. Circular RNAs and gene fusion within the esophageal transcriptome. Figure S4. Saliva and esophageal microbiota in the early stages of the EAC cascade. Figure S5. Esophageal microbiota in the early stages of the EAC cascade. Figure S6. Procrustes analysis between transcriptome and microbiome. Figure S7. Correlations between Campylobacter iOTU36 and transcript counts. Figure S8. Prevalence of regions of interest in shotgun metagenomics data.

\section{Acknowledgements}

The authors would like to thank all patients who participated in this study and staff of the Gastrointestinal and Liver Unit, The Prince of Wales Hospital (Sydney) for their help with recruitment.

\section{Authors' contributions}

Conceptualization, N.O.K.; Methodology, N.P.D., S.M.R., T.L.R., C.C.O., S.M.M., N.T., N.O.K.; Formal analysis, N.P.D., S.N., N.O.K.; Investigation, C.J.G., C.C.O., B.S.M.F., S.A.W., N.C.R., N.O.K.; Resources, S.A.W., S.M.R., M.R.W., N.O.K.; Data curation, N.P.D., N.O.K.; Writing - Original Draft, N.O.K.; Writing - Review \& Editing, N.P.D., S.M.R., T.L.R., C.C.O., N.C.R., S.M.M., N.T., M.R.W.; Visualization, N.P.D., N.O.K.; Supervision, N.T., N.C.R., N.O.K.; Funding Acquisition, S.M.R., M.R.W., N.O.K. All authors read and approved the final manuscript.

\section{Funding}

This work was supported by a Cancer Institute NSW Career Development Fellowship (15/CDF/1-11 to N.O.K.) and a UNSW Scientia fellowship. MRW and NPD acknowledge funding from the Australian Federal Government NCRIS Scheme, the New South Wales State Government RAAP Scheme and the University of New South Wales. NCR is supported by a Cancer Institute NSW Early Career Fellowship (2019/ECF1082) and a UNSW Scientia fellowship.

\section{Availability of data and materials}

The PacBio 16S rRNA amplicon sequencing data (https://www.ebi.ac.uk/ena/ browser/view/PRJEB46879) [80], the Illumina 16S rRNA amplicon sequencing data (https://www.ebi.ac.uk/ena/browser/view/PRJEB46880) [81] and bacterial genome assemblies (https://www.ebi.ac.uk/ena/browser/view/PRJEB46877) [82] were submitted to the European Nucleotide Archive (ENA). The RNA sequencing data from esophageal epithelial cells was also submitted to ENA (https://www.ebi.ac.uk/ena/browser/view/PRJEB46795) [83]. The RNA sequencing data from esophageal biopsies are not publicly available to maintain patient confidentiality but are available from the corresponding author on reasonable request. In-house scripts for the identification of possible functional consequences of alternative splicing events were submitted to zenodo (https://doi.org/10.5281/zenodo.5179440) [21]. The data from the previous cohort profiling esophageal brushings is available from ENA (16S rRNA amplicon: https:/www.ebi.ac.uk/ena/browser/view/PRJEB25236; shotgun metagenomics: https://www.ebi.ac.uk/ena/browser/view/PRJEB25422) [10].

\section{Declarations}

\section{Ethics approval and consent to participate}

Ethics approval for the prospectively recruited cohort was obtained from the South Eastern Sydney Local Health District Human Research Ethics Committee (HREC 16/020). Data from the previously published cohort was approved through the South Eastern Sydney Local Health District Human Research Ethics Committee (HREC 13/375). All subjects recruited to the study signed a written informed consent. Growth of primary macrophages was approved by the University of New South Wales Human Research Ethics Committee (HC14203 for 2018 and 2019 and HC190417 for 2021). All research conformed to the principles of the Declaration of Helsinki.

\section{Consent for publication}

Not applicable

\section{Competing interests}

The authors declare that they have no competing interests.

\section{Author details}

${ }^{1}$ School of Biotechnology and Biomolecular Sciences, UNSW Sydney, Sydney, NSW 2052, Australia. ${ }^{2}$ Gastrointestinal and Liver Unit, The Prince of Wales Hospital, Randwick, NSW 2031, Australia. ${ }^{3}$ School of Medical Sciences, Faculty 
of Medicine, UNSW Sydney, Sydney, NSW 2052, Australia. ${ }^{4}$ Ramaciotti Centre for Genomics, UNSW Sydney, Sydney, NSW 2052, Australia. ${ }^{5}$ School of Women's and Children's Health, UNSW Sydney, Sydney, NSW 2052, Australia. ${ }^{6}$ The John Curtin School of Medical Research, The Australian National University, Canberra, ACT 2601, Australia.

\section{Received: 29 January 2021 Accepted: 11 August 2021}

Published online: 19 August 2021

\section{References}

1. Arnold M, Abnet CC, Neale RE, Vignat J, Giovannucci EL, McGlynn KA, et al. Global burden of 5 major types of gastrointestinal cancer. Gastroenterology. 2020;159(1):335-49 e15. https://doi.org/10.1053/j.gastro.2020.02.068.

2. He H, Chen N, Hou Y, Wang Z, Zhang Y, Zhang G, et al. Trends in the incidence and survival of patients with esophageal cancer: A SEER database analysis. Thorac Cancer. 2020;11(5):1121-8. https://doi.org/10.1111/17597714.13311.

3. Januszewicz W, Fitzgerald RC. Barrett's oesophagus and oesophageal adenocarcinoma. Medicine (Abingdon). 2019;47:275-85.

4. Tan MC, Mansour N, White DL, Sisson A, El-Serag HB, Thrift AP. Systematic review with meta-analysis: prevalence of prior and concurrent Barrett's oesophagus in oesophageal adenocarcinoma patients. Aliment Pharmacol Ther. 2020;52(1):20-36. https://doi.org/10.1111/apt.15760.

5. GBD 2017 Gastro-oesophageal Reflux Disease Collaborators. The global, regional, and national burden of gastro-oesophageal reflux disease in 195 countries and territories, 1990-2017: a systematic analysis for the Global Burden of Disease Study 2017. Lancet Gastroenterol Hepatol. 2020;5:561-81.

6. Lopetuso LR, Severgnini M, Pecere S, Ponziani FR, Boskoski I, Larghi A, et al. Esophageal microbiome signature in patients with Barrett's esophagus and esophageal adenocarcinoma. PLoS One. 2020;15(5):e0231789. https://doi. org/10.1371/journal.pone.0231789.

7. Nardone G, Compare D, Rocco A. A microbiota-centric view of diseases of the upper gastrointestinal tract. Lancet Gastroenterol Hepatol. 2017;2(4): 298-312. https://doi.org/10.1016/S2468-1253(16)30108-X.

8. Di J, Cheng Y, Chang D, Liu Y. A meta-analysis of the impact of obesity, metabolic syndrome, insulin resistance, and microbiome on the diagnosis of Barrett's esophagus. Dig Dis. 2020;38(3):165-77. https://doi.org/10.1159/ 000502376.

9. Munch NS, Fang HY, Ingermann J, Maurer HC, Anand A, Kellner V, et al. High-fat diet accelerates carcinogenesis in a mouse model of Barrett's esophagus via interleukin 8 and alterations to the gut microbiome. Gastroenterology. 2019;157(2):492-506 e2. https://doi.org/10.1053/j.gastro.2 019.04.013.

10. Deshpande NP, Riordan SM, Castano-Rodriguez N, Wilkins MR, Kaakoush NO. Signatures within the esophageal microbiome are associated with host genetics, age, and disease. Microbiome. 2018;6(1):227. https://doi.org/10.11 86/s40168-018-0611-4.

11. Kaakoush NO, Castano-Rodriguez N, Man SM, Mitchell HM. Is Campylobacter to esophageal adenocarcinoma as Helicobacter is to gastric adenocarcinoma? Trends Microbiol. 2015;23(8):455-62. https://doi.org/10.101 6/j.tim.2015.03.009

12. Lloyd-Price J, Arze C, Ananthakrishnan AN, Schirmer M, Avila-Pacheco J, Poon TW, et al. Multi-omics of the gut microbial ecosystem in inflammatory bowel diseases. Nature. 2019;569(7758):655-62. https://doi.org/10.1038/s41 586-019-1237-9.

13. Lundell LR, Dent J, Bennett JR, Blum AL, Armstrong D, Galmiche JP, et al. Endoscopic assessment of oesophagitis: clinical and functional correlates and further validation of the Los Angeles classification. Gut. 1999;45(2):17280. https://doi.org/10.1136/gut.45.2.172.

14. Patro R, Duggal G, Love MI, Irizarry RA, Kingsford C. Salmon provides fast and bias-aware quantification of transcript expression. Nat Methods. 2017; 14(4):417-9. https://doi.org/10.1038/nmeth.4197.

15. Newman AM, Steen CB, Liu CL, Gentles AJ, Chaudhuri AA, Scherer F, et al. Determining cell type abundance and expression from bulk tissues with digital cytometry. Nat Biotechnol. 2019;37(7):773-82. https://doi.org/10.1038/ s41587-019-0114-2.

16. Love MI, Huber W, Anders S. Moderated estimation of fold change and dispersion for RNA-seq data with DESeq2. Genome Biol. 2014;15(12):550. https://doi.org/10.1186/s13059-014-0550-8.

17. Kuleshov MV, Jones MR, Rouillard AD, Fernandez NF, Duan Q, Wang Z, et al. Enrichr: a comprehensive gene set enrichment analysis web server 2016 update. Nucleic Acids Res. 2016;44(W1):W90-7. https://doi.org/10.1093/nar/ gkw377.

18. Shen S, Park JW, Lu ZX, Lin L, Henry MD, Wu YN, et al. rMATS: robust and flexible detection of differential alternative splicing from replicate RNA-Seq data. Proc Natl Acad Sci U S A. 2014;111(51):E5593-601. https://doi.org/10.1 073/pnas.1419161111.

19. Sterne-Weiler T, Weatheritt RJ, Best AJ, Ha KCH, Blencowe BJ. Efficient and accurate quantitative profiling of alternative splicing patterns of any complexity on a laptop. Mol Cell. 2018;72(1):187-200 e6. https://doi.org/10.1 016/j.molcel.2018.08.018.

20. Lin KT, Krainer AR. PSI-Sigma: a comprehensive splicing-detection method for short-read and long-read RNA-seq analysis. Bioinformatics. 2019;35(23): 5048-54. https://doi.org/10.1093/bioinformatics/btz438.

21. Deshpande NP, Kaakoush NO. Scripts to identify possible functional changes due to alternative splicing. Zenodo. https://doi.org/10.5281/ zenodo.5179440 (2021).

22. Veiga DFT. maser: Mapping Alternative Splicing Events to pRoteins. R package version 170. 2020

23. Brennan P. drawProteins: a Bioconductor/R package for reproducible and programmatic generation of protein schematics. F1000Res. 2018;7:1105.

24. Mi H, Muruganujan A, Ebert D, Huang X, Thomas PD. PANTHER version 14 more genomes, a new PANTHER GO-slim and improvements in enrichment analysis tools. Nucleic Acids Res. 2019;47(D1):D419-D26. https://doi.org/10.1 093/nar/gky1038.

25. Zhang $X O$, Dong $R$, Zhang $Y$, Zhang JL, Luo Z, Zhang J, et al. Diverse alternative back-splicing and alternative splicing landscape of circular RNAs. Genome Res. 2016;26(9):1277-87. https://doi.org/10.1101/gr.202 895.115.

26. Haas BJ, Dobin A, Li B, Stransky N, Pochet N, Regev A. Accuracy assessment of fusion transcript detection via read-mapping and de novo fusion transcript assembly-based methods. Genome Biol. 2019;20(1):213. https:// doi.org/10.1186/s13059-019-1842-9.

27. Kozich JJ, Westcott SL, Baxter NT, Highlander SK, Schloss PD. Development of a dual-index sequencing strategy and curation pipeline for analyzing amplicon sequence data on the MiSeq Illumina sequencing platform. Appl Environ Microbiol. 2013;79(17):5112-20. https://doi.org/10.1128/AEM.01 043-13.

28. Schloss PD, Westcott SL, Ryabin T, Hall JR, Hartmann M, Hollister EB, et al. Introducing mothur: open-source, platform-independent, communitysupported software for describing and comparing microbial communities. Appl Environ Microbiol. 2009;75(23):7537-41. https://doi.org/10.1128/AEM.01 541-09.

29. Segata N, Izard J, Waldron L, Gevers D, Miropolsky L, Garrett WS, et al. Metagenomic biomarker discovery and explanation. Genome Biol. 2011; 12(6):R60. https://doi.org/10.1186/gb-2011-12-6-r60.

30. Knights D, Kuczynski J, Charlson ES, Zaneveld J, Mozer MC, Collman $R G$, et al. Bayesian community-wide culture-independent microbial source tracking. Nat Methods. 2011;8(9):761-3. https://doi.org/10.1038/ nmeth.1650.

31. Feng K, Zhang Z, Cai W, Liu W, Xu M, Yin H, et al. Biodiversity and species competition regulate the resilience of microbial biofilm community. Mol Ecol. 2017;26(21):6170-82. https://doi.org/10.1111/mec.14356.

32. Reshef DN, Reshef YA, Finucane HK, Grossman SR, McVean G, Turnbaugh PJ, et al. Detecting novel associations in large data sets. Science. 2011; 334(6062):1518-24. https://doi.org/10.1126/science.1205438.

33. Mitchell A, Rentero C, Endoh Y, Hsu K, Gaus K, Geczy C, et al. LILRA5 is expressed by synovial tissue macrophages in rheumatoid arthritis, selectively induces pro-inflammatory cytokines and IL-10 and is regulated by TNF-alpha, IL-10 and IFN-gamma. Eur J Immunol. 2008;38(12):3459-73. https://doi.org/10.1002/eji.200838415.

34. Kaakoush NO, Deshpande NP, Wilkins MR, Tan CG, Burgos-Portugal JA Raftery MJ, et al. The pathogenic potential of Campylobacter concisus strains associated with chronic intestinal diseases. PLoS One. 2011;6(12):e29045. https://doi.org/10.1371/journal.pone.0029045.

35. Cox MP, Peterson DA, Biggs PJ. SolexaQA: At-a-glance quality assessment of Illumina second-generation sequencing data. BMC Bioinformatics. 2010; 11(1):485. https://doi.org/10.1186/1471-2105-11-485.

36. Koren S, Walenz BP, Berlin K, Miller JR, Bergman NH, Phillippy AM. Canu: scalable and accurate long-read assembly via adaptive k-mer weighting and repeat separation. Genome Res. 2017;27(5):722-36. https://doi.org/10.1101/ gr.215087.116. 
37. Walker BJ, Abeel T, Shea T, Priest M, Abouelliel A, Sakthikumar S, et al. Pilon: an integrated tool for comprehensive microbial variant detection and genome assembly improvement. PLoS One. 2014;9(11):e112963. https://doi. org/10.1371/journal.pone.0112963.

38. Seemann T. Prokka: rapid prokaryotic genome annotation. Bioinformatics. 2014;30(14):2068-9. https://doi.org/10.1093/bioinformatics/btu153.

39. Lee I, Ouk Kim Y, Park SC, Chun J. OrthoANI: An improved algorithm and software for calculating average nucleotide identity. Int J Syst Evol Microbiol. 2016;66(2):1100-3. https://doi.org/10.1099/ijsem.0.000760.

40. Petkau A, Stuart-Edwards M, Stothard P, Van Domselaar G. Interactive microbial genome visualization with GView. Bioinformatics. 2010;26(24): 3125-6. https://doi.org/10.1093/bioinformatics/btq588.

41. Lechner M, Findeiss S, Steiner L, Marz M, Stadler PF, Prohaska SJ. Proteinortho: detection of (co-)orthologs in large-scale analysis. BMC Bioinformatics. 2011;12(1):124. https://doi.org/10.1186/1471-2105-12-124.

42. El Mortaji L, Aubert S, Galtier E, Schmitt C, Anger K, Redko Y, et al. The Sole DEAD-Box RNA Helicase of the Gastric Pathogen Helicobacter pylori Is Essential for Colonization. mBio. 2018;9(2):e02071-17.

43. Benoit SL, Maier RJ. Site-directed mutagenesis of Campylobacter concisus respiratory genes provides insight into the pathogen's growth requirements. Sci Rep. 2018;8(1):14203. https://doi.org/10.1038/s41598-01 8-32509-9.

44. Park M, Raftery MJ, Thomas PS, Geczy CL, Bryant K, Tedla N. Leukocyte immunoglobulin-like receptor B4 regulates key signalling molecules involved in FcgammaRI-mediated clathrin-dependent endocytosis and phagocytosis. Sci Rep. 2016;6(1):35085. https://doi.org/10.1038/srep35085.

45. Kaakoush NO, Deshpande NP, Man SM, Burgos-Portugal JA, Khattak FA, Raftery MJ, et al. Transcriptomic and proteomic analyses reveal key innate immune signatures in the host response to the gastrointestinal pathogen Campylobacter concisus. Infect Immun. 2015;83(2):832-45. https://doi.org/1 0.1128/IAl.03012-14.

46. Man SM, Kaakoush NO, Leach ST, Nahidi L, Lu HK, Norman J, et al. Host attachment, invasion, and stimulation of proinflammatory cytokines by Campylobacter concisus and other non-Campylobacter jejuni Campylobacter species. J Infect Dis. 2010;202(12):1855-65. https://doi.org/10.1086/657316.

47. Vandamme P, Falsen E, Pot B, Hoste B, Kersters K, De Ley J. Identification of EF group 22 campylobacters from gastroenteritis cases as Campylobacter concisus. J Clin Microbiol. 1989;27(8):1775-81. https://doi.org/10.1128/jcm.27. 8.1775-1781.1989.

48. Needham BD, Trent MS. Fortifying the barrier: the impact of lipid A remodelling on bacterial pathogenesis. Nat Rev Microbiol. 2013;11(7):46781. https://doi.org/10.1038/nrmicro3047

49. Wang X, Kim Y, Ma Q, Hong SH, Pokusaeva K, Sturino JM, et al. Cryptic prophages help bacteria cope with adverse environments. Nat Commun. 2010;1(1):147. https://doi.org/10.1038/ncomms1146.

50. Wang X, Kim Y, Wood TK. Control and benefits of CP4-57 prophage excision in Escherichia coli biofilms. ISME J. 2009;3(10):1164-79. https://doi.org/10.103 8/ismej.2009.59.

51. Sethi D, Mahajan S, Singh C, Lama A, Hade MD, Gupta P, et al. Lipoprotein Lprl of Mycobacterium tuberculosis acts as a lysozyme inhibitor. J Biol Chem. 2016;291(6):2938-53. https://doi.org/10.1074/jbc.M115.662593.

52. Iwata T, Watanabe A, Kusumoto M, Akiba M. Peptidoglycan acetylation of Campylobacter jejuni is essential for maintaining cell wall integrity and colonization in chicken intestines. Appl Environ Microbiol. 2016;82(20):628490. https://doi.org/10.1128/AEM.02068-16.

53. Moons LM, Kusters JG, Bultman E, Kuipers EJ, van Dekken H, Tra WM, et al. Barrett's oesophagus is characterized by a predominantly humoral inflammatory response. J Pathol. 2005;207(3):269-76. https://doi.org/10.1 002/path. 1847.

54. Kanoh S, Tanabe T, Rubin BK. IL-13-induced MUC5AC production and goblet cell differentiation is steroid resistant in human airway cells. Clin Exp Allergy. 2011;41(12):1747-56. https://doi.org/10.1111/j.1365-2222.2011.03852.x.

55. Zhu Z, Homer RJ, Wang Z, Chen Q, Geba GP, Wang J, et al. Pulmonary expression of interleukin-13 causes inflammation, mucus hypersecretion, subepithelial fibrosis, physiologic abnormalities, and eotaxin production. J Clin Invest. 1999;103(6):779-88. https://doi.org/10.1172/JCl5909.

56. Fisher OM, Levert-Mignon AJ, Lord SJ, Botelho NK, Freeman AK, Thomas ML, et al. High expression of cathepsin E in tissues but not blood of patients with Barrett's esophagus and adenocarcinoma. Ann Surg Oncol. 2015;22(7): 2431-8. https://doi.org/10.1245/s10434-014-4155-y.
57. Pelaseyed T, Hansson GC. CFTR anion channel modulates expression of human transmembrane mucin MUC3 through the PDZ protein GOPC. J Cell Sci. 2011;124(18):3074-83. https://doi.org/10.1242/jcs.076943.

58. Timmer JR, Mak TW, Manova K, Anderson KV, Niswander L. Tissue morphogenesis and vascular stability require the Frem2 protein, product of the mouse myelencephalic blebs gene. Proc Natl Acad Sci U S A. 2005: 102(33):11746-50. https://doi.org/10.1073/pnas.0505404102.

59. Wang L, Gu J, Liu R, Mao W, Xu F, Liu Z, et al. FRAS1-related extracellular matrix protein 2 drives the progression and poor prognosis of IL-1 $\beta$ associated esophageal squamous cell carcinoma. Research Square. 2019; doi:https://doi.org/10.21203/rs.2.19017/v1.

60. Bakowski MA, Desjardins CA, Smelkinson MG, Dunbar TL, Lopez-Moyado IF, Rifkin SA, et al. Ubiquitin-mediated response to microsporidia and virus infection in C. elegans. PLoS Pathog. 2014;10(6):e1004200.

61. Nishishita K, Sakai E, Okamoto K, Tsukuba T. Structural and phylogenetic comparison of napsin genes: the duplication, loss of function and humanspecific pseudogenization of napsin B. Gene. 2013;517(2):147-57. https://doi. org/10.1016/j.gene.2013.01.013.

62. Kaakoush NO, Mitchell HM. Campylobacter concisus - a new player in intestinal disease. Front Cell Infect Microbiol. 2012;2:4.

63. Polk DB, Peek RM Jr. Helicobacter pylori: gastric cancer and beyond. Nat Rev Cancer. 2010;10(6):403-14. https://doi.org/10.1038/nrc2857.

64. Spilker T, Uluer AZ, Marty FM, Yeh WW, Levison JH, Vandamme $P$, et al. Recovery of Herbaspirillum species from persons with cystic fibrosis. J Clin Microbiol. 2008;46(8):2774-7. https://doi.org/10.1128/JCM.00460-08.

65. Santos LS, Antunes CA, Santos CS, Pereira JA, Sabbadini PS, Luna M, et al. Corynebacterium diphtheriae putative tellurite-resistance protein (CDCE8392 0813) contributes to the intracellular survival in human epithelial cells and lethality of Caenorhabditis elegans. Mem Inst Oswaldo Cruz. 2015;110(5):6628. https://doi.org/10.1590/0074-02760140479.

66. Turner RJ, Taylor DE, Weiner JH. Expression of Escherichia coli TehA gives resistance to antiseptics and disinfectants similar to that conferred by multidrug resistance efflux pumps. Antimicrob Agents Chemother. 1997; 41(2):440-4. https://doi.org/10.1128/AAC.41.2.440.

67. Nejman D, Livyatan I, Fuks G, Gavert N, Zwang Y, Geller LT, et al. The human tumor microbiome is composed of tumor type-specific intracellular bacteria. Science. 2020;368(6494):973-80. https://doi.org/10.1126/science.aay9189.

68. Warren RL, Freeman DJ, Pleasance S, Watson P, Moore RA, Cochrane K, et al. Co-occurrence of anaerobic bacteria in colorectal carcinomas. Microbiome. 2013;1(1):16. https://doi.org/10.1186/2049-2618-1-16.

69. Sudhakar JN, Lu HH, Chiang HY, Suen CS, Hwang MJ, Wu SY, et al. Lumenal Galectin-9-Lamp2 interaction regulates lysosome and autophagy to prevent pathogenesis in the intestine and pancreas. Nat Commun. 2020;11(1):4286. https://doi.org/10.1038/s41467-020-18102-7.

70. Dall $\mathrm{E}$, Brandstetter $\mathrm{H}$. Structure and function of legumain in health and disease. Biochimie. 2016;122:126-50. https://doi.org/10.1016/j.biochi.2015. 09.022.

71. Pires D, Bernard EM, Pombo JP, Carmo N, Fialho C, Gutierrez MG, et al. Mycobacterium tuberculosis modulates miR-106b-5p to control cathepsin S expression resulting in higher pathogen survival and poor T-cell activation. Front Immunol. 2017:8:1819. https://doi.org/10.3389/fimmu.2017.01819.

72. Memmert S, Damanaki A, Nogueira AVB, Eick S, Nokhbehsaim M, Papadopoulou AK, et al. Role of cathepsin S in periodontal inflammation and infection. Mediators Inflamm. 2017;2017:4786170.

73. Aubry C, Goulard C, Nahori MA, Cayet N, Decalf J, Sachse M, et al. OatA, a peptidoglycan O-acetyltransferase involved in Listeria monocytogenes immune escape, is critical for virulence. J Infect Dis. 2011;204(5):731-40. https://doi.org/10.1093/infdis/jir396.

74. Kim KH, An DR, Song J, Yoon JY, Kim HS, Yoon HJ, et al. Mycobacterium tuberculosis Eis protein initiates suppression of host immune responses by acetylation of DUSP16/MKP-7. Proc Natl Acad Sci U S A. 2012;109(20):772934. https://doi.org/10.1073/pnas.1120251109.

75. Maag JLV, Fisher OM, Levert-Mignon A, Kaczorowski DC, Thomas ML, Hussey DJ, et al. Novel aberrations uncovered in Barrett's esophagus and esophageal adenocarcinoma using whole transcriptome sequencing. Mol Cancer Res. 2017;15(11):1558-69. https://doi.org/10.11 58/1541-7786.MCR-17-0332.

76. Park SY, Kim JY, Lee SM, Chung JO, Lee KH, Jun CH, et al. Expression of early growth response gene-1 in precancerous lesions of gastric cancer. Oncol Lett. 2016;12(4):2710-5. https://doi.org/10.3892/ol.2016.4962. 
77. Wu MY, Liang YR, Wu XY, Zhuang CX. Relationship between Egr-1 gene expression and apoptosis in esophageal carcinoma and precancerous lesions. World J Gastroenterol. 2002;8(6):971-5. https://doi.org/10.3748/wjg. v8.6.971.

78. Buckley AM, Lynam-Lennon N, Kennedy SA, Dunne MR, Aird JJ, Foley EK, et al. Leukaemia inhibitory factor is associated with treatment resistance in oesophageal adenocarcinoma. Oncotarget. 2018;9(72):33634-47. https://doi. org/10.18632/oncotarget.25950.

79. Burrell RA, McClelland SE, Endesfelder D, Groth P, Weller MC, Shaikh N, et al. Replication stress links structural and numerical cancer chromosomal instability. Nature. 2013;494(7438):492-6. https://doi.org/10.1038/nature1 1935.

80. Deshpande NP, Riordan SM, Gorman CJ, Nielsen S, Russell TL, Correa-Ospina C, et al. Multi-omics of the esophageal microenvironment identifies signatures associated with progression of Barrett's esophagus. BioProject PRJEB46879. European Nucleotide Archive, 2021. https://www.ebi.ac.uk/ena/ browser/view/PRJEB46879

81. Deshpande NP, Riordan SM, Gorman CJ, Nielsen S, Russell TL, Correa-Ospina $C$, et al. Multi-omics of the esophageal microenvironment identifies signatures associated with progression of Barrett's esophagus. BioProject PRJEB46880. European Nucleotide Archive, 2021. https://www.ebi.ac.uk/ena/ browser/view/PRJEB46880

82. Deshpande NP, Riordan SM, Castaño-Rodríguez N, Wilkins MR, Kaakoush NO. Signatures within the esophageal microbiome are associated with host genetics, age, and disease. BioProject PRJEB25236. European Nucleotide Archive, 2018. https://www.ebi.ac.uk/ena/browser/view/PRJEB25236

83. Deshpande NP, Riordan SM, Gorman CJ, Nielsen S, Russell TL, Correa-Ospina $C$, et al. Multi-omics of the esophageal microenvironment identifies signatures associated with progression of Barrett's esophagus. BioProject PRJEB46795. European Nucleotide Archive, 2021. https://www.ebi.ac.uk/ena/ browser/view/PRJEB46795

\section{Publisher's Note}

Springer Nature remains neutral with regard to jurisdictional claims in published maps and institutional affiliations.

Ready to submit your research? Choose BMC and benefit from:

- fast, convenient online submission

- thorough peer review by experienced researchers in your field

- rapid publication on acceptance

- support for research data, including large and complex data types

- gold Open Access which fosters wider collaboration and increased citations

- maximum visibility for your research: over $100 \mathrm{M}$ website views per year

At $\mathrm{BMC}$, research is always in progress.

Learn more biomedcentral.com/submissions 